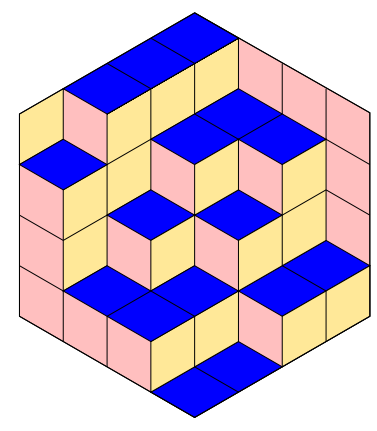

ALGEBRAIC COMBINATORICS

Emily Barnard, Andrew Carroll \& Shijie Zhu

Minimal inclusions of torsion classes

Volume 2, issue 5 (2019), p. 879-901.

<http://alco.centre-mersenne.org/item/ALCO_2019__2_5_879_0>

(c) The journal and the authors, 2019.

Some rights reserved.

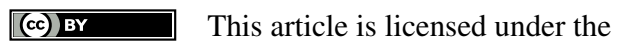

Creative Commons ATtribution 4.0 International LiCEnSE.

http://creativecommons.org/licenses/by/4.0/

Access to articles published by the journal Algebraic Combinatorics on the website http://alco.centre-mersenne.org/ implies agreement with the Terms of Use (http://alco.centre-mersenne.org/legal/).

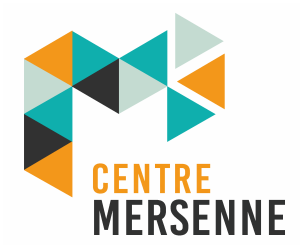

Algebraic Combinatorics is member of the Centre Mersenne for Open Scientific Publishing www.centre-mersenne.org 


\title{
Minimal inclusions of torsion classes
}

\author{
Emily Barnard, Andrew Carroll \& Shijie Zhu
}

\begin{abstract}
Let $\Lambda$ be a finite-dimensional associative algebra. The torsion classes of $\bmod \Lambda$ form a lattice under containment, denoted by tors $\Lambda$. In this paper, we characterize the cover relations in tors $\Lambda$ by certain indecomposable modules. We consider three applications: First, we show that the completely join-irreducible torsion classes (torsion classes which cover precisely one element) are in bijection with bricks. Second, we characterize faces of the canonical join complex of tors $\Lambda$ in terms of representation theory. Finally, we show that, in general, the algebra $\Lambda$ is not characterized by its lattice tors $\Lambda$. In particular, we study the torsion theory of a quotient of the preprojective algebra of type $A_{n}$. We show that its torsion class lattice is isomorphic to the weak order on $A_{n}$.
\end{abstract}

\section{INTRODUCTION}

Let $\Lambda$ be a finite-dimensional associative algebra over a field $k$, and write tors $\Lambda$ for the set of torsion classes of finitely generated modules over $\Lambda$, partially ordered by containment. The poset tors $\Lambda$ is a complete lattice in which the meet (or greatest lower bound) $\bigwedge\left\{\mathcal{T}, \mathcal{T}^{\prime}\right\}$ coincides with the intersection $\mathcal{T} \cap \mathcal{T}^{\prime}$, and the join (or smallest upper bound) $\bigvee\left\{\mathcal{T}, \mathcal{T}^{\prime}\right\}$ coincides with the iterative extension closure of the union $\mathcal{T} \cup$ $\mathcal{T}^{\prime}$. In this paper, we study the cover relations $\mathcal{T}^{\prime}>\mathcal{T}$ in tors $\Lambda$. Recall that a torsion class $\mathcal{T}^{\prime}$ covers $\mathcal{T}$ if $\mathcal{T} \subsetneq \mathcal{T}^{\prime}$ and for each $\mathcal{Y} \in$ tors $\Lambda$, if $\mathcal{T} \subsetneq \mathcal{Y} \subseteq \mathcal{T}^{\prime}$ then $\mathcal{Y}=\mathcal{T}^{\prime}$.

In [1], the authors describe the lattice of functorially-finite torsion classes by way of $\tau$-tilting pairs. They show the existence of a unique module which encodes each cover relation as follows: When $\mathcal{T}$ and $\mathcal{T}^{\prime}$ are functorially finite torsion classes, with $\mathcal{T}^{\prime}>\mathcal{T}$, there exists a unique module $M$ with the property that $\mathcal{T}^{\prime}$ is the closure of $\operatorname{add}(\mathcal{T} \cup\{M\})$ under taking quotients.

In our complimentary approach, we show that for each cover relation $\mathcal{T}^{\prime}>\mathcal{T}$ in tors $\Lambda$, there exists a unique "minimal" module $M$ with the property that $\mathcal{T}^{\prime}$ is the closure of $\mathcal{T} \cup\{M\}$ under taking iterative extensions. Below, we make the notation of "minimal" precise.

Definition 1.1. A module $M$ is a minimal extending module for $\mathcal{T}$ if it satisfies the following three properties:

(P1) Every proper factor of $M$ is in $\mathcal{T}$;

(P2) If $0 \rightarrow M \rightarrow X \rightarrow T \rightarrow 0$ is a non-split exact sequence with $T \in \mathcal{T}$, then $X \in \mathcal{T}$

(P3) $\operatorname{Hom}(\mathcal{T}, M)=0$.

Manuscript received 18th May 2018, revised 14th January 2019, accepted 6th March 2019.

KEYWORDS. lattice theory, torsion classes, canonical join representations. 
In general, a minimal extending module $M$ is a quotient of the module studied in [1]. For a more precise description of this relationship see [2, Proposition 1.9], [10, Theorem 4.1], and [11, Theorem 3.11].

The following remarks will be useful. Assuming Property (P1), Property (P3) is equivalent to the fact that $M \notin \mathcal{T}$. Observe that if there is a non-trivial homomorphism from a module in $\mathcal{T}$ to $M$, then both the image and cokernel are in $\mathcal{T}$, and $M$ is an extension of the cokernel by the image. Thus, any minimal extending module is indecomposable. Indeed, direct summands are proper factors, and torsion classes are closed under direct sums.

In the statement of the following theorem, and throughout the paper we have the following notation: We write $[M]$ for the isoclass of the module $M ; \operatorname{ME}(\mathcal{T})$ for the set of isoclasses $[M]$ such that $M$ is a minimal extending module for $\mathcal{T}$; and $\mathscr{F} i l t(\mathcal{T} \cup\{M\})$ for the iterative extension closure of $\mathcal{T} \cup\{M\}$.

THEOREM 1.2. Let $\mathcal{T}$ be a torsion class over $\Lambda$. Then the map

$$
\eta_{\mathcal{T}}:[M] \mapsto \mathscr{F} \operatorname{ilt}(\mathcal{T} \cup\{M\})
$$

is a bijection from the set $\operatorname{ME}(\mathcal{T})$ to the set of $\mathcal{T}^{\prime} \in$ tors $\Lambda$ such that $\mathcal{T} \lessdot \mathcal{T}^{\prime}$.

Recall that a module $M$ is called a brick if the endomorphism ring of $M$ is a division ring. (That is, the non-trivial endomorphisms are invertible.)

THEOREM 1.3. Let $\Lambda$ be a finite-dimensional associative algebra and $M \in \bmod \Lambda$. Then $M$ is a minimal extending module for some torsion class if and only if $M$ is a brick.

As an immediate consequence of Theorem 1.3 we obtain a labeling of cover relations for tors $\Lambda$ (when they exist) by bricks. Independently and concurrently with our work, the authors of [11] also give a labeling of the cover relations in the lattice of torsion classes by brick modules which coincides with the labeling proposed here. We illustrate in Example 3.2 that in tors $\Lambda$ there exists pairs $\mathcal{T}^{\prime}>\mathcal{T}$ such that there is no torsion class $\mathcal{Y}$ satisfying $\mathcal{T}^{\prime}>\mathcal{Y} \geqslant \mathcal{T}$.

This paper fits into a larger body of research which studies the combinatorial structure of the lattice of (functorially finite) torsion classes. Connections between the combinatorics of a finite simply laced Weyl group $W$ and the corresponding preprojective algebra $\Pi W$ are of particular interest. In [15], Mizuno showed the lattice of functorially finite torsion classes f-tors $\Pi W$ is isomorphic to the weak order on $W$. Building on this work, the authors of [13] have shown that the lattice of (functorially finite) torsion classes of quotients of $\Pi W$ are lattice quotients of the weak order on $W$. They also obtain an analogous labeling of f-tors $\Pi W$ by certain modules called layer modules. (See [13, Theorem 1.3].) Cover relations in the lattice of torsion classes are also closely related to maximal green sequences [6]. In a related direction, the authors of [12] study certain lattice properties of f-tors $\Lambda$ when $\Lambda$ arises from a quiver that is mutation-equivalent to a path quiver or oriented cycle, including a certain minimal "factorization" called the canonical join representation.

Inspired by these results, we will give three applications of Theorems 1.2 and 1.3. Before describing them, we recall some terminology in lattice theory. In a (not necessarily finite) lattice $L$, a join representation for an element $w \in L$ is an expression $\bigvee A=w$, where $A$ is a (possibly infinite) subset of $L$. An element $w$ is join-irreducible, if $w \in A$ for any join representation $w=\bigvee A$, where $A$ is a finite set. An element $w$ is completely join-irreducible, if $w \in A$ for any join representation $w=\bigvee A$, where $A$ is any subset of $L$. (By convention, the smallest element in $L$ is not join-irreducible or completely join-irreducible.) Equivalently, $w$ is completely join-irreducible if and only if $w$ covers only one element $v$ and any element $u<w \in L$ satisfies $u \leqslant v$. 
EXAmPle 1.4. Let $L$ be the lattice of $\mathbb{N} \cup\{\infty\}$ with natural partial order. Then $\infty$ is join-irreducible but not completely join-irreducible.

For our first application, we have the following theorem; see [12, Lemma 8.2], [13, Theorems 1.1 and 1.2], and [11, Theorem 3.3] for analogous results. (In the statement below $\operatorname{Gen}(M)$ is the closure of $M$ under taking factors and direct sums.)

Theorem 1.5. Suppose that $M$ is an indecomposable $\Lambda$ module. Then the map $\zeta$ : $[M] \mapsto \mathscr{F} i l t(\operatorname{Gen}(M))$ is a bijection from the set of isoclasses of bricks over $\Lambda$ to the set of of completely join-irreducible elements in tors $\Lambda$.

For our second application, we consider the canonical join representation in tors $\Lambda$. A join representation $\bigvee A$ is irredundant if $\bigvee A^{\prime}<\bigvee A$ for each subset $A^{\prime} \subseteq A$. Informally, the canonical join representation of $w$ is the unique lowest irredundant join representation $\bigvee A$ for $w$, when such an expression exists. In this case, we also say that the set $A$ is a canonical join representation. (We make the notion "lowest" precise in Section 3.2.) In particular, each element $a \in A$ is join-irreducible.

EXAMPLE 1.6. Consider the top element $\hat{1}$ in the pentagon lattice $N_{5}$ shown on the left in Figure 1. Its irredundant join representations are $\bigvee \hat{1}, \bigvee\{x, z\}$ and $\bigvee\{x, y\}$ Observe that $\bigvee\{x, y\}$ is "lower" than $\bigvee\{x, z\}$ because $x \leqslant z$ (and $y \leqslant y$ ). Indeed, $\bigvee\{x, y\}$ is the canonical join representation of $\hat{1}$.

It is natural to ask which collections $A$ of join-irreducible elements in $L$ satisfy $\bigvee A$ is a canonical join representation. The collection of such subsets, which we denote by $\Gamma(L)$, has the structure of an abstract simplicial complex whose vertex set is the set of join-irreducible elements in $L$. (See, for instance, [16, Proposition 2.2], for the case when $L$ is finite.) We call $\Gamma(L)$ the canonical join complex of $L$.
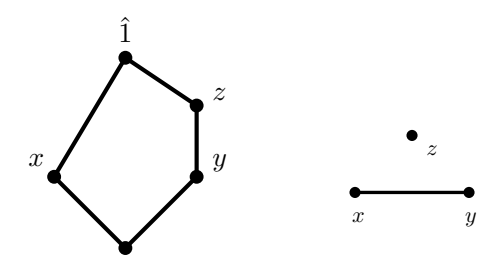

Figure 1. A lattice $N_{5}$ and its canonical join complex $\Gamma\left(N_{5}\right)$.

EXAmple 1.7. Consider the pentagon lattice $N_{5}$ shown on the left in Figure 1. The canonical join complex $\Gamma\left(N_{5}\right)$ appears on the right. The set $\{x, z\} \notin \Gamma\left(N_{5}\right)$ because $\bigvee\{x, z\}=\hat{1}$ is not the canonical join representation for $\hat{1}$, and $\{y, z\}$ is not a face in $\Gamma\left(N_{5}\right)$ because $\bigvee\{y, z\}$ is not even irredundant.

Our second application characterizes the collections of subsets $A$ of completely join-irreducible elements that belong to $\Gamma(\operatorname{tors} \Lambda)$. For the second statement of the theorem below, recall that tors $\Lambda$ is finite if and only if $\Lambda$ is $\tau$-rigid finite (and in this case tors $\Lambda$ is equal to the lattice of functorially finite torsion classes f-tors $\Lambda$ ). See [14] and Remark 3.8.

THEOREM 1.8. Suppose that $\mathcal{E}$ is a collection of bricks over $\Lambda$. Then the set $\{\mathscr{F}$ ilt $(\operatorname{Gen} M): M \in \mathcal{E}\}$ is a face of $\Gamma(\operatorname{tors} \Lambda)$ if and only if each pair $M$ and $M^{\prime}$ satisfies the compatibility condition known as hom-orthogonality:

$$
\operatorname{dim} \operatorname{Hom}_{\Lambda}\left(M, M^{\prime}\right)=\operatorname{dim} \operatorname{Hom}_{\Lambda}\left(M^{\prime}, M\right)=0 .
$$

In particular, if $\Lambda$ is $\tau$-rigid finite then $\Gamma$ (tors $\Lambda$ ) is isomorphic to the complex of hom-orthogonal $\Lambda$ brick modules. 
A collection of hom-orthogonal bricks were also studied in [2], where they are called semibricks.

Finally, we consider the question: Is the algebra $\Lambda$ characterized by its lattice of torsion classes? For our third application, we give an extended counter-example. We study the torsion theory of a certain quotient of the preprojective algebra in type $A_{n}$, which we call $R A_{n}$. Unlike the preprojective algebra, $R A_{n}$ has finite representation type for each $n$. We describe the canonical join complex of $R A_{n}$, and construct an explicit isomorphism from tors $R A_{n}$ to the weak order on $A_{n}$. Thus, by Mizuno's result, $R A_{n}$ shares the same torsion theory as $\Pi A_{n}$.

\section{Minimal inClusions AMONG TORSiOn ClASSES}

In this section, we prove Theorems 1.2 and 1.3. In Proposition 2.7, we verify that the map $\eta_{\mathcal{T}}$ from Theorem 1.2 is well-defined. That is, we argue that $\mathscr{F} i l t(\mathcal{T} \cup\{M\})$ is indeed a torsion class, and that it covers $\mathcal{T}$. In Theorem 2.8 we construct an inverse map of $\eta_{\mathcal{T}}$. The proof of the forward direction of Theorem 1.3 can be found in Lemma 2.3 while the remaining direction appears in the proof of Proposition 2.13.

2.1. Preliminaries. Throughout, we take $\Lambda$ to be a finite-dimensional associative algebra over a field $k$, and we write $\bmod \Lambda$ for the category of finite-dimensional (left) modules over $\Lambda$. For $\mathcal{T}$ a class of modules over $\Lambda$ (which we assume to be closed under isomorphism), we write ind $\mathcal{T}$ for the set of indecomposable modules $M \in \mathcal{T}$ and [ind $\mathcal{T}]$ for the set of isoclasses $[M]$ such that $M \in$ ind $\mathcal{T}$.

A torsion class $\mathcal{T}$ is a class of modules that is closed under factors, isomorphisms, and extensions. Dually, a class of modules $\mathcal{F}$ is a torsion-free class if it is closed under submodules, isomorphisms, and extensions. As in the introduction, tors $\Lambda$ denotes the lattice of torsion classes over $\Lambda$, in which $\mathcal{T} \leqslant \mathcal{T}^{\prime}$ if and only if $\mathcal{T} \subseteq \mathcal{T}^{\prime}$. We write torf $\Lambda$ for the lattice of torsion-free classes also ordered by containment.

At times it will be useful to translate a result or a proof from the language of torsion classes to the language of torsion-free classes. To do this, we make use of the following standard dualities. Given a torsion class $\mathcal{T}$, denote by $\mathcal{T}^{\perp}$ the set of modules $X \in \bmod \Lambda$ such that $\operatorname{Hom}_{\Lambda}(\mathcal{T}, X)=0$. The map $(-)^{\perp}:$ tors $\Lambda \rightarrow$ torf $\Lambda$ is a poset anti-isomorphim with inverse given by

$$
\mathcal{F} \mapsto^{\perp} \mathcal{F}:=\left\{X \in \bmod \Lambda: \operatorname{Hom}_{\Lambda}(X, \mathcal{F})=0\right\} .
$$

The duality functor $D=\operatorname{Hom}_{k}(-, k): \bmod \Lambda \rightarrow \bmod \Lambda^{\mathrm{op}}$ also furnishes a poset antiisomorphism from tors $\Lambda$ to torf $\Lambda^{\text {op }}$ with inverse given by the same duality. (Recall that $\Lambda^{\text {op }}$ denotes the opposite algebra of $\Lambda$. For details, see [14] or [17].)

Let $\mathcal{S}$ be a set of indecomposable modules in $\bmod \Lambda$. An $\mathcal{S}$-filtration (of length $l$ ) of a module $M$ in $\bmod \Lambda$ is a sequence of submodules

$$
M=M_{l} \supsetneq M_{l-1} \supsetneq \cdots \supsetneq M_{1} \supsetneq M_{0}=0
$$

such that $M_{i} / M_{i-1}$ is isomorphic to a module in $\mathcal{S}$ for each $i=1, \ldots, l$. We write $\mathscr{F} i l t^{(l)}(\mathcal{S})$ for the class of modules $M$ that admit an $\mathcal{S}$-filtration of length at most $l$ and $\mathscr{F}$ ilt $(\mathcal{S})$ for the class of all modules in $\bmod \Lambda$ that admit an $\mathcal{S}$-filtration, i.e. $\mathscr{F}$ ilt $(\mathcal{S})=$ $\bigcup_{l \geqslant 0} \mathscr{F} i l t^{(l)}(\mathcal{S})$. When $\mathcal{S}$ is not a class of indecomposable modules (but rather an additive full subcategory, for instance) we abuse the notation and write $\mathscr{F} i l t(\mathcal{S})$ instead of $\mathscr{F} i l t($ ind $\mathcal{S})$. We close this subsection with two lemmas.

Lemma 2.1. Let $\mathcal{S}$ be a class of indecomposable modules in $\bmod \Lambda$. The class $\mathscr{F} i l t(\mathcal{S})$ is closed under extensions. 
Proof. Suppose there is an exact sequence

$$
0 \rightarrow N \rightarrow M \stackrel{\pi}{\rightarrow} N^{\prime} \rightarrow 0
$$

with $N \in \mathscr{F} i l t^{(l)}(\mathcal{S})$ and $N^{\prime} \in \mathscr{F} i l t^{\left(l^{\prime}\right)}(\mathcal{S})$.

Let $N=N_{l} \supsetneq N_{l-1} \supsetneq \ldots \supsetneq N_{0}=0$ and $N^{\prime}=N_{l^{\prime}}^{\prime} \supsetneq N_{l^{\prime}-1}^{\prime} \supsetneq \ldots \supsetneq N_{0}^{\prime}=0$ be an $\mathcal{S}$-filtration of $N$ and $N^{\prime}$ respectively. Take $M_{i}$ to be the pull back following, for $0 \leqslant i \leqslant l^{\prime}$ :

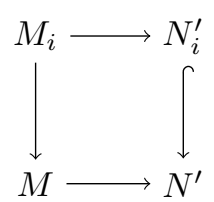

Then $M=M_{l^{\prime}} \supsetneq M_{l^{\prime}-1} \supsetneq \cdots M_{0}=N=N_{l} \supsetneq N_{l-1} \supsetneq \cdots \supsetneq N_{0}=0$ is an $\mathcal{S}$-filtration of $M$.

Lemma 2.2. Suppose that $\mathcal{S}$ is a class of indecomposable modules that is closed under taking indecomposable summands of factors. Then $\mathscr{F}$ ilt $(\mathcal{S})$ is closed under factors. Dually, if $\mathcal{S}$ is closed under taking indecomposable summands of submodules, then $\mathscr{F}$ ilt $(\mathcal{S})$ is closed under submodules.

Proof. Suppose that $N \in \mathscr{F} i l t^{(l)}(\mathcal{S})$ and $\phi: N \rightarrow U$ is an epimorphism. We assume, without loss of generality, that $U$ is indecomposable and proceed by induction on l. If $l=1$, then $N \in \mathcal{S}$. Since $\mathcal{S}$ is closed under epimorphisms, we have $U \in \mathcal{S}$. Now suppose that all factors of modules in $\mathscr{F} i l t^{\left(l^{\prime}\right)}(\mathcal{S})$ are in $\mathscr{F} i l t(\mathcal{S})$ for $l^{\prime}<l$. Since $N \in \mathscr{F} i l t^{(l)}(\mathcal{S})$, it admits an $\mathcal{S}$-filtration $N=N_{l} \supsetneq N_{l-1} \supsetneq \cdots \supsetneq N_{0}=0$ with $N_{i} / N_{i-1}$ isomorphic to an object in $\mathcal{S}$. Consider the submodule $\phi\left(N_{l-1}\right)$ of $U$, and notice that $\phi\left(N_{l-1}\right)$ is a factor of $N_{l-1} \in \mathscr{F} i l t^{(l-1)}(\mathcal{S})$. Therefore, by induction, $\phi\left(N_{l-1}\right)$ has an $\mathcal{S}$-filtration. Further, $U / \phi\left(N_{l-1}\right)$ is a factor of $N_{l} / N_{l-1} \in \mathcal{S}$. Therefore $U / \phi\left(N_{l-1}\right)$ belongs to $\mathcal{S}$. We have the following short exact sequence:

$$
0 \rightarrow \phi\left(N_{l-1}\right) \rightarrow U \stackrel{\pi}{\rightarrow} U / \phi\left(N_{l-1}\right) \rightarrow 0 .
$$

Lemma 2.1 then implies that $U$ is in $\mathscr{F} i l t(\mathcal{S})$, as desired.

2.2. Minimal extending Modules. In this subsection, we prove Theorem 1.2. We begin by showing that $\eta_{\mathcal{T}}:[M] \mapsto \mathscr{F} i l t(\mathcal{T} \cup\{M\})$ is well-defined. The first statement in the next lemma confirms that $\mathscr{F} i l t(\mathcal{T} \cup\{M\})$ is indeed a torsion class. Because it follows immediately, we also dispense with the forward direction of Theorem 1.3.

Lemma 2.3. Suppose that $\mathcal{T} \in$ tors $\Lambda$ and $M$ is an indecomposable $\Lambda$ module that does not belong to $\mathcal{T}$. If every proper factor of $M$ lies in $\mathcal{T}$ then:

(1) $\mathscr{F} i l t(\mathcal{T} \cup\{M\})$ is a torsion class and

(2) $M$ is a brick over $\Lambda$.

Proof. The first statement follows immediately from Lemma 2.2. To prove the second statement, let $f: M \rightarrow M$ be a non-zero morphism, and assume that $f$ is not an isomorphism. Consider the exact sequence:

$$
0 \rightarrow \operatorname{Im} f \rightarrow M \rightarrow M / \operatorname{Im} f \rightarrow 0 .
$$

Observe that both $\operatorname{Im} f$ and $M / \operatorname{Im} f$ are proper factors of $M$, and thus belong to $\mathcal{T}$. Because $\mathcal{T}$ is closed under extensions, it follows that $M \in \mathcal{T}$, and that is a contradiction. Therefore $\operatorname{End}(M)$ only contains automorphisms and hence $\operatorname{End}(M)$ is a division ring. So $M$ is a brick. 
EXAMPLE 2.4. In light of Lemma 2.3, we might be tempted to think that condition (P1) is sufficient for constructing cover relations in tors $\Lambda$. For a nonexample, consider the torsion class $\mathcal{T}=\left\{3,{ }_{2}^{3}\right\}$ in tors $k Q$ for the quiver $Q=3 \longrightarrow 2 \longrightarrow 1$. (We write $i$ for the simple at $i$, and ${ }_{2}^{3}$ for the representation $k \stackrel{1}{\longrightarrow} k \longrightarrow 0$.) The simple module at vertex 1 trivially satisfies the condition that all of its proper factors belong to $\mathcal{T}$. We have the following chain of torsion classes in tors $\Lambda$ :

$$
\mathcal{T}=\operatorname{add}\left\{3,{ }_{2}^{3}\right\} \subsetneq \text { add }\left\{3,{ }_{2}^{3}, \underset{1}{2}\right\} \subsetneq \text { add }\left\{3,{ }_{2}^{3}, \underset{1}{3}, 1\right\}=\mathscr{F} i l t(\mathcal{T} \cup\{1\}) .
$$

Below (Proposition 2.6) we establish precisely when $\mathscr{F} i l t(\mathcal{T} \cup\{M\}$ ) covers $\mathcal{T}$.

LEMma 2.5. Let $\mathcal{T} \in$ tors $\Lambda$ and $M \notin \mathcal{T}$ be indecomposable such that each proper factor of $M$ belongs to $\mathcal{T}$. Let $N \in \mathscr{F}$ ilt $(\mathcal{T} \cup\{M\}) \backslash \mathcal{T}$ such that each proper factor of $N$ lies in $\mathcal{T}$. If $\mathscr{F}$ ilt $(\mathcal{T} \cup\{M\})>\mathcal{T}$ then $N \cong M$.

Proof. Write $\mathcal{T}^{\prime}$ for $\mathscr{F}$ ilt $(\mathcal{T} \cup\{M\})$. Note that $N$ also satisfies the assumptions of Lemma 2.3, so $\mathscr{F}$ ilt $(\mathcal{T} \cup\{N\}$ ) is a torsion class which properly contains $\mathcal{T}$ (since $N \notin \mathcal{T})$ and is contained in $\mathcal{T}^{\prime}$. Hence, $\mathcal{T}^{\prime}=\mathscr{F} i l t(\mathcal{T} \cup\{N\})$. In particular, $N$ admits a $\mathcal{T} \cup\{M\}$-filtration with at least one subfactor isomorphic to $M$, so $\operatorname{dim} N \geqslant \operatorname{dim} M$. Symmetrically, $M$ admits a $\mathcal{T} \cup\{N\}$-filtration with at least one subfactor isomorphic to $N$. Therefore, $M \cong N$.

Proposition 2.6. Let $\mathcal{T} \in$ tors $\Lambda$ and $M \notin \mathcal{T}$ be an indecomposable module such that each proper factor of $M$ belongs to $\mathcal{T}$. Then $\mathcal{T}^{\prime}=\mathscr{F}$ ilt $(\mathcal{T} \cup\{M\})>\mathcal{T}$ if and only if $M$ is a factor of each $N \in \mathcal{T}^{\prime} \backslash \mathcal{T}$.

Proof. First we argue the "if" direction. Suppose that every element in $\mathcal{T}^{\prime} \backslash \mathcal{T}$ admits an epimorphism to $M$. Let $\mathcal{G}$ be a torsion class with $\mathcal{T} \subsetneq \mathcal{G} \subseteq \mathcal{T}^{\prime}$, and pick $N \in \mathcal{G} \backslash \mathcal{T}$. Because $M$ is a factor of $N$, we have $M \in \mathcal{G}$. Thus, $\mathcal{T}^{\prime}=\mathscr{F} i l t(\mathcal{T} \cup\{M\}) \subseteq \mathcal{G}$, as desired.

Conversely, suppose that $\mathcal{T}^{\prime}>\mathcal{T}$, and let $N$ be any module in $\mathcal{T}^{\prime} \backslash \mathcal{T}$. Among all submodules $N^{\prime} \subseteq N$ such that $N / N^{\prime} \in \mathcal{T}^{\prime} \backslash \mathcal{T}$, choose $N^{\prime}$ maximal. By Lemma 2.5 $N / N^{\prime}$ is isomorphic to $M$. We conclude $M$ is a factor of $N$.

We use the previous proposition to verify that $\eta_{\mathcal{T}}$ (from Theorem 1.2) has the correct codomain. In the next proposition, $\operatorname{cov}^{\uparrow}(\mathcal{T})$ denotes the set of torsion classes $\mathcal{T}^{\prime}$ such that $\mathcal{T}^{\prime} \rightarrow \mathcal{T}$.

Proposition 2.7. Suppose that $\mathcal{T} \in$ tors $\Lambda$ and $M$ is an indecomposable $\Lambda$ module. If $M$ is a minimal extending module for $\mathcal{T}$, then $\mathscr{F} i l t(\mathcal{T} \cup\{M\})>\mathcal{T}$. That is, the map $\eta_{\mathcal{T}}: \operatorname{ME}(\mathcal{T}) \rightarrow \operatorname{cov}^{\uparrow}(\mathcal{T})$ is well-defined.

Proof. Again, denote by $\mathcal{T}^{\prime}$ the torsion class $\mathscr{F} i l t(\mathcal{T} \cup\{M\})$. Properties $(\mathrm{P} 1)$ and $(\mathrm{P} 3)$ imply that $M$ satisfies the hypotheses of Proposition 2.6. Let $N \in \mathcal{T}^{\prime} \backslash \mathcal{T}$ with ind $\mathcal{T} \cup\{M\}$-filtration $N=N_{l} \supsetneq N_{l-1} \supsetneq \cdots \supsetneq N_{0}=0$. Assume, without loss of generality, that $N$ is indecomposable. We $\operatorname{argue}$ by induction on $l$ that $M$ is a factor of $N$. If $l=1$, then $N \cong M$ since $N \notin \mathcal{T}$. Suppose that $l>1$, and let $i$ to be the smallest index such that $N_{i} / N_{i-1} \cong M$ (one must exist since $N \notin \mathcal{T}$ ).

If $i=1$, then there is a short exact sequence

$$
0 \rightarrow M \rightarrow N \rightarrow N / N_{1} \rightarrow 0 .
$$

Since $N$ was assumed indecomposable (and $l>1$ ), the sequence does not split. If $N / N_{1} \in \mathcal{T}$, then by Property (P2), $N \in \mathcal{T}$, a contradiction. Otherwise, $N / N_{1} \notin \mathcal{T}$ is in $\mathscr{F} i t^{(l-1)}(\mathcal{T} \cup\{M\})$, so by induction, $M$ is a factor of $N / N_{1}$, so it is also a factor of $N$.

If $i>1$, consider the short exact sequence

$$
0 \rightarrow N_{i-1} \rightarrow N \rightarrow N / N_{i-1} \rightarrow 0
$$


which again is non-split by assumption. Note that since $N_{i-1}$ has a filtration by modules in $\mathcal{T}, N_{i-1} \in \mathcal{T}$. If $N / N_{i-1} \in \mathcal{T}$, then so is $N$, since torsion classes are closed under extensions. Therefore, $N / N_{i-1} \notin \mathcal{T}$, and it has a $\mathcal{T} \cup\{M\}$-filtration of length $l-i+1<l$. Hence, by induction, $M$ is a factor of $N / N_{i-1}$, and therefore of $N$ as well.

Hence, $M$ is a factor of $N$ for each module $N$ in $\mathcal{T}^{\prime}$, so $\mathcal{T} \lessdot \mathcal{T}^{\prime}$.

Below we construct the inverse map to $\eta_{\mathcal{T}}$, completing the proof of Theorem 1.2. Recall that [ind $\Lambda]$ is the set of isoclasses $[M]$ such that $M \in$ ind $\Lambda$.

THEOREM 2.8. For each $\mathcal{T}^{\prime} \rightarrow \mathcal{T}$ in tors $\Lambda$, there exists a unique (up to isomorphism) indecomposable module $M$ such that $\mathcal{T}^{\prime}=\mathscr{F} i l t(\mathcal{T} \cup\{M\})$. Furthermore, the map $\mathscr{F}$ ilt $(\mathcal{T} \cup\{M\}) \mapsto[M]$ is the inverse to $\eta_{\mathcal{T}}$.

Proof of Theorem 2.8 and Theorem 1.2. Let $N \in \mathcal{T}^{\prime} \backslash \mathcal{T}$. As in the proof of Proposition 2.6, among all submodules $N^{\prime}$ of $N$ such that $N / N^{\prime} \in \mathcal{T}^{\prime} \backslash \mathcal{T}$, choose $N^{\prime}$ maximal. We take $M$ to be the factor $N / N^{\prime}$. (It is immediate that $M$ is indecomposable.) Because $\mathcal{T}^{\prime}>\mathcal{T}$, we conclude that $\mathcal{T}^{\prime}=\mathscr{F} i l t(\mathcal{T} \cup\{M\})$. Lemma 2.5 implies that $M$ is unique up to isomorphism.

To prove the second statement, it is enough to show that $M$ is a minimal extending module. It is immediate that $M$ satisfies Property (P1). Suppose that $0 \rightarrow M \stackrel{i}{\rightarrow} X \stackrel{\pi}{\rightarrow}$ $T \rightarrow 0$ is a short exact sequence with $T \in \mathcal{T}$. If $X \in \mathcal{T}^{\prime} \backslash \mathcal{T}$, then Proposition 2.6 implies that there is an epimorphism $q: X \rightarrow M$. If $q \circ i=0$, then $q$ factors through $\pi$ which implies that $M$ is a quotient of $T$, contradicting with $M \notin \mathcal{T}$. If $q \circ i \neq 0$, then $q \circ i$ is an isomorphism due to the fact that $M$ is a brick. So the exact sequence splits. Thus $M$ satisfies property (P2). By construction, $M \notin \mathcal{T}$. Thus according to the first remark after Definition 1.1, $M$ satisfies (P3).

The following corollary will be useful as we explore the connection to the canonical join complex of tors $\Lambda$ in Section 3.2.

Corollary 2.9. Let $\mathcal{T}_{1}$ and $\mathcal{T}_{2}$ be distinct torsion classes in tors $\Lambda$, and for each $i \in\{1,2\}$, let $M_{i}$ be a minimal extending module for $\mathcal{T}_{i}$. If $\mathscr{F} i l t\left(\mathcal{T}_{1} \cup\left\{M_{1}\right\}\right)=\mathscr{F}$ ilt $\left(\mathcal{T}_{2} \cup\right.$ $\left.\left\{M_{2}\right\}\right)$ then

$$
\operatorname{dim} \operatorname{Hom}_{\Lambda}\left(M_{1}, M_{2}\right)=\operatorname{dim} \operatorname{Hom}_{\Lambda}\left(M_{2}, M_{1}\right)=0 .
$$

Proof. Write $\mathcal{T}^{\prime}$ for $\mathscr{F}$ ilt $\left(\mathcal{T}_{1} \cup\left\{M_{1}\right\}\right)=\mathscr{F}$ ilt $\left(\mathcal{T}_{2} \cup\left\{M_{2}\right\}\right)$. First, we claim that $M_{1}$ belongs to $\mathcal{T}_{2}$ and $M_{2}$ belongs to $\mathcal{T}_{1}$. Then the statement follow immediately from Property (P3) of minimal extending modules. Since $\mathcal{T}_{1}$ and $\mathcal{T}_{2}$ are distinct and both covered by the same torsion class $\mathcal{T}^{\prime}$, it follows that there exists some $N \in \mathcal{T}_{1} \backslash \mathcal{T}_{2}$. In particular, $N \in \mathcal{T}^{\prime} \backslash \mathcal{T}_{2}$. Proposition 2.6 implies that $N$ surjects onto $M_{2}$. Thus, $M_{2} \in \mathcal{T}_{1}$. By symmetry $M_{1} \in \mathcal{T}_{2}$.

2.3. TORSION-FREE ClASSES AND BRICKS. We now record the corresponding notions for torsion-free classes, both for completeness, and for a convenient proposition relating the upper covers of $\mathcal{T}$ to the lower covers of $\mathcal{T}^{\perp}$. (See Proposition 2.12 below.) Proofs that are essentially equivalent to their counterparts in the torsion context are suppressed. We begin by defining the analogue to minimally extending modules.

Definition 2.10. A module $M$ is called a minimal co-extending module for a torsionfree class $\mathcal{F}$ if it satisfies the following three conditions:

$\left(\mathrm{P} 1^{\prime}\right)$ Every proper submodule of $M$ is in $\mathcal{F}$;

$\left(\mathrm{P}^{\prime}\right)$ if $0 \rightarrow F \rightarrow X \rightarrow M \rightarrow 0$ is a non-split exact sequence, with $F \in \mathcal{F}$, then $X \in \mathcal{F}$

$\left(\mathrm{P} 3^{\prime}\right) \operatorname{Hom}_{\Lambda}(M, \mathcal{F})=0$. 
In Theorem 2.11 below, the notation $\operatorname{cov}^{\uparrow}(\mathcal{F})$ is the set of torsion-free classes $\mathcal{F}^{\prime}$ such that $\mathcal{F}^{\prime} \rightarrow \mathcal{F}$.

THEOREM 2.11. Suppose that $\mathcal{F}$ is a torsion-free class over $\Lambda$ and $M \notin \mathcal{F}$ is an indecomposable $\Lambda$ module. The following are equivalent:

(1) $M$ is a minimal co-extending module of $\mathcal{F}$.

(2) Each proper submodule of $M$ lies in $\mathcal{F}$ and $M$ is a submodule for each $N \in$ $\mathscr{F}$ ilt $(\mathcal{F} \cup\{M\}) \backslash \mathcal{F}$.

(3) $\mathscr{F}$ ilt $(\mathcal{F} \cup\{M\})>\mathcal{F}$.

The map $\zeta:[M] \mapsto \mathscr{F}$ ilt $(\mathcal{F} \cup\{M\})$ is a bijection from the set of isoclasses $[M]$ such that $M$ is a minimal co-extending module for $\mathcal{F}$ to the $\operatorname{set} \operatorname{cov}^{\uparrow}(\mathcal{F})$.

Proposition 2.12. Suppose that $\mathcal{T}$ is a torsion class in tors $\Lambda$ and $M$ is an indecomposable $\Lambda$ module such that $\mathscr{F}$ ilt $(\mathcal{T} \cup\{M\})$ is a torsion class. Then $M$ is a minimal extending module for the torsion class $\mathcal{T}$ if and only if it is a minimal co-extending module for the torsion-free class $\mathscr{F}$ ilt $(\mathcal{T} \cup\{M\})^{\perp}$.

Proof. We argue the forward implication of the proposition; the reverse implication is similar. Let $\mathcal{T}^{\prime}$ denote the torsion class $\eta_{\mathcal{T}}([M])=\mathscr{F}$ ilt $(\mathcal{T} \cup\{M\})$. Observe that $M \notin\left(\mathcal{T}^{\prime}\right)^{\perp}$. Also, Property (P3) implies that $M$ belongs to $\mathcal{T}^{\perp}$. By Theorem 2.11, it is enough to show that $M$ satisfies the following: First, each proper submodule of $M$ belongs to $\left(\mathcal{T}^{\prime}\right)^{\perp}$; and second, $M$ is a submodule of each $X \in \mathcal{T}^{\perp} \backslash\left(\mathcal{T}^{\prime}\right)^{\perp}$.

Suppose that $M^{\prime}$ is an indecomposable submodule of $M$, and $N$ belongs to $\mathcal{T}^{\prime} \backslash \mathcal{T}$. We claim that $\operatorname{Hom}_{\Lambda}\left(N, M^{\prime}\right)$ is nonzero only if $M^{\prime}=M$. Let $\phi: N \rightarrow M^{\prime}$ be such a nonzero homomorphism. On one hand, $\operatorname{Im} \phi \in \mathcal{T}^{\prime}$ (because $\mathcal{T}^{\prime}$ is closed under epimorphisms). On the other hand, $\operatorname{Im} \phi \in \mathcal{T}^{\perp}$ (because torsion-free classes are closed under submodules). In particular, $\operatorname{Im} \phi \notin \mathcal{T}$. By Proposition 2.6, there is a surjection of $\operatorname{Im} \phi$ onto $M$. Because $\operatorname{Im} \phi$ is a submodule of $M$, we obtain $\operatorname{Im} \phi \cong M^{\prime} \cong M$, as desired. We conclude that each proper submodule of $M$ belongs to $\left(\mathcal{T}^{\prime}\right)^{\perp}$.

Suppose that $X \in \mathcal{T}^{\perp} \backslash\left(\mathcal{T}^{\prime}\right)^{\perp}$, and let $f: N \rightarrow X$ be a nonzero morphism from a module $N \in \mathcal{T}^{\prime} \backslash \mathcal{T}$. We may assume that $f$ is injective. (If it is not injective, then replace $N$ with $N / \operatorname{ker}(f)$.) We claim that $M$ is a submodule of $N$. Let $N=N_{l} \supsetneq$ $\cdots \supsetneq N_{0}=0$ be an $\operatorname{ind}(\mathcal{T}) \cup\{M\}$-filtration of $N$. Since $N \notin \mathcal{T}$, there is some index $i$ such that $N_{i} / N_{i-1}$ is isomorphic to $M$. For the moment, assume that $i>1$, so that $N_{1} \in \mathcal{T}$. Then we have a nonzero homomorphism $N_{1} \hookrightarrow N \hookrightarrow X$ from a module in $\mathcal{T}$ to $X$. That is a contradiction. Thus, $M \cong N_{1} \hookrightarrow N \hookrightarrow X$ as desired.

We close this section by completing the proof of Theorem 1.3. Recall that in Lemma 2.3, we showed that if $M$ is a minimal extending module, then it is a brick (the forward implication in Theorem 1.3). By Proposition 2.12 it is enough to show: If $M$ is a brick, then there exists a torsion-free class $\mathcal{F}$ such that $M$ is a minimal co-extending module for $\mathcal{F}$. Also recall that $\mathscr{F} i l t(\operatorname{Gen} M)$ is always a torsion class.

Proposition 2.13. If $M$ is a brick over $\Lambda$, then $M$ is a minimal co-extending module for $\mathscr{F} i l t(\operatorname{Gen} M)^{\perp}$.

Proof of Proposition 2.13 and Theorem 1.3. Suppose that $M$ is a brick over $\Lambda$. We will argue that $M$ is a minimal co-extending module for the torsion-free class $\mathcal{F}=$ $\mathscr{F} i l t(\operatorname{Gen} M)^{\perp}$.

First, we claim that $\mathscr{F} i l t(\operatorname{Gen} M)^{\perp}=M^{\perp}:=\left\{X: \operatorname{Hom}_{\Lambda}(M, X)=0\right\}$. It is immediate that $\mathscr{F} i l t(\operatorname{Gen} M)^{\perp} \subseteq M^{\perp}$. Conversely, let $X \in M^{\perp}$. Since $\operatorname{Hom}(-, X)$ is left exact, it follows that $\operatorname{Hom}(L, X) \hookrightarrow \operatorname{Hom}(\oplus M, X)=0$ for each epimorphism $\oplus M \rightarrow L$. Hence $X \in \operatorname{Gen}(M)^{\perp}$. Then using induction on the length of a filtration 
of $N$, for all $N \in \mathscr{F} i l t(\operatorname{Gen}(M))$, it is easy to see $\operatorname{Hom}(N, X)=0$. Hence $X$ is in $\mathscr{F}$ ilt $(\operatorname{Gen}(M))^{\perp}$. Therefore Property $\left(\mathrm{P} 3^{\prime}\right)$ holds.

To verify $\left(\mathrm{P} 1^{\prime}\right)$, let $M^{\prime}$ be a proper submodule of $M$. If $\operatorname{Hom}_{\Lambda}\left(M, M^{\prime}\right) \neq 0$, then $M$ is not a brick, a contradiction. Thus, $M^{\prime} \in\{X: \operatorname{Hom}(M, X)=0\}=\mathscr{F} i l t(\operatorname{Gen} M)^{\perp}$.

To verify $\left(\mathrm{P}^{\prime}\right)$, suppose that there is a non-split short exact sequence

$$
0 \rightarrow F \stackrel{f}{\rightarrow} X \stackrel{g}{\rightarrow} M \rightarrow 0
$$

with $F \in \mathcal{F}$. If $X \notin \mathcal{F}$, then there is a nonzero homomorphism $\pi: M \rightarrow X$. Since $M$ is a brick, and $g \circ \pi$ is a endomorphism of $M$ (which is not an isomorphism since the sequence is non-split), $g \circ \pi=0$. Hence, $\pi$ factors through $f$. Since $\operatorname{Hom}_{\Lambda}(M, F)=0$, we have a contradiction.

Therefore, $M$ is a minimal co-extending module for $\mathcal{F}$. The statement follows from Proposition 2.12.

\section{Applichtions}

We consider two applications of Theorems 1.2 and 1.3. First, in Proposition 3.1, we prove that there is a bijective correspondence between isoclasses $[M]$ of bricks over $\Lambda$ and completely join-irreducible torsion classes in tors $\Lambda$ via the map sending $[M]$ to $\mathscr{F} i l t(\operatorname{Gen}(M))$. Second, we consider the canonical join complex of tors $\Lambda$, proving the forward implication of Theorem 1.8 in Proposition 3.5, and we completing the proof in Proposition 3.7.

3.1. Completely Join-IRreduCible torsion Classes. In this section, we prove Theorem 1.5 as Proposition 3.1 below. Recall that $\mathcal{T}$ is completely join-irreducible if for each (possibly infinite) subset $A \subseteq$ tors $\Lambda, \mathcal{T}=\bigvee A$ implies that $\mathcal{T} \in A$. Equivalently, $\mathcal{T}$ is completely join-irreducible if and only if it covers precisely one torsion class $\mathcal{S}$, and $\mathcal{G} \subseteq \mathcal{S}$ for any torsion class $\mathcal{G} \subsetneq \mathcal{T}$. In the statement below, recall that Gen $M$ denotes the factor-closure of add $M$.

Proposition 3.1. The torsion class $\mathcal{T}$ is completely join-irreducible if and only if there exists a brick $M$ such that $\mathcal{T}$ is equal to $\mathscr{F}$ ilt $(\operatorname{Gen} M)$. In this case, the brick $M$ is unique up to isomorphism. In particular, the map $\zeta:[M] \mapsto \mathscr{F}$ ilt $(\operatorname{Gen} M)$ from Theorem 1.5 is a bijection.

Proof Proposition 3.1 and Theorem 1.5. Suppose that the torsion class $\mathcal{T}$ is completely join-irreducible and write $\mathcal{S}$ for the unique torsion class covered by $\mathcal{T}$. Theorem 1.3 implies that there exists a unique (up to isomorphism) brick $M$ such that $\mathcal{T}=\mathscr{F}$ ilt $(\mathcal{S} \cup\{M\})$. We claim that $\mathcal{T}=\mathscr{F}$ ilt $(\operatorname{Gen} M)$. Observe that $\mathscr{F}$ ilt $($ Gen $M)$ is contained in $\mathcal{T}$, and $\mathscr{F} i l t($ Gen $M) \nsubseteq \mathcal{S}$ (because $M \notin \mathcal{S}$ ). The claim follows.

Conversely, let $M$ be a brick over $\Lambda$. In the proof of Proposition 2.13, we showed that $M$ is a minimal co-extending module for $\mathscr{F} i l t(\operatorname{Gen} M)^{\perp}$. By Proposition 2.12, there exists a torsion class $\mathcal{S}$ such that $\mathscr{F} i l t(\operatorname{Gen} M)>\mathcal{S}$ and $M$ is a minimal extending module for $\mathcal{S}$. (That is $\mathscr{F}$ ilt $(\operatorname{Gen} M)=\mathscr{F} i l t(\mathcal{S} \cup\{M\})$ ).) Suppose that $\mathcal{G} \subseteq \mathscr{F}$ ilt $(\mathrm{Gen} M)$. If $\mathcal{G} \nsubseteq \mathcal{S}$ then there exists some module $N \in \mathcal{G} \backslash \mathcal{S}$. In particular, $N \in \mathscr{F} i l t(\operatorname{Gen} M) \backslash \mathcal{S}$. Proposition 2.6 says that $M$ is a factor of $N$, hence $M \in \mathcal{G}$. We conclude that $\mathcal{G}=\mathscr{F} i l t($ Gen $M)$. Thus any torsion class $\mathcal{G} \subsetneq \mathscr{F} i l t(\operatorname{Gen} M)$ also satisfies $\mathcal{G} \subseteq \mathcal{S}$.

We conclude that $\mathscr{F} i l t(\operatorname{Gen} M)$ is completely join-irreducible.

REMARK 3.2. There exist join-irreducible torsion classes which are not completely joinirreducible. Consider $\bmod k Q$ where $Q$ is the Kronecker quiver and $k$ is algebraically closed (see Figure 2). 


$$
1 \underset{b}{\stackrel{a}{\rightleftharpoons}} 2
$$

FiguRE 2. The Kronecker quiver.

Let $n$ be a non-negative integer, and write $V_{n}$ for the representation defined as follows: $V_{n}(1)=k^{n+1}, V_{n}(2)=k^{n} ; V_{n}(a)=\left[\begin{array}{ll}I_{n} & \mathbf{0}\end{array}\right]$ and $V_{n}(b)=\left[\begin{array}{ll}\mathbf{0} & I_{n}\end{array}\right]$ where $I_{n}$ is the $n \times n$ identity matrix, and $\mathbf{0}$ is a column of zeros. (The module corresponding to each $V_{n}$ is indecomposable and preinjective; see e.g. [4, VIII.1].) Let $\mathcal{I}_{n}$ denote the additive closure of $\left\{V_{0}, V_{1}, \ldots, V_{n}\right\}$, and $\mathcal{I}_{\infty}=\bigcup_{n \geqslant 0} \mathcal{I}_{n}$. It is an easy exercise to verify that both $\mathcal{I}_{n}$ and $\mathcal{I}_{\infty}$ are torsion classes and $\mathcal{I}_{n} \lessdot \mathcal{I}_{n+1}<\mathcal{I}_{\infty}$ for each $n$. Observe that $\mathcal{I}_{\infty}$ is join-irreducible, but not completely join-irreducible. In particular, it does not cover any elements in tors $k Q$. Each brick in $\mathcal{I}_{\infty}$ is isomorphic to $V_{n}$ for some $n \geqslant 0$, and it can be shown that $\mathcal{I}_{\infty}$ cannot be expressed as $\mathcal{I}_{n}$ for any such $n$.

3.2. The CANONiCAL JOIN COMPleX of tors $\Lambda$. In this section, we characterize certain faces of the canonical join complex of tors $\Lambda$. Before we begin, we review the necessary lattice-theoretic terminology. Recall that a lattice $L$ is a poset such that, for each finite subset $A \subseteq L$, the join or least upper bound $\bigvee A$ exists and, dually, the meet or greatest lower bound $\bigwedge A$ exists. The lattice tors $\Lambda$ is a complete lattice, meaning that $\bigvee A$ and $\bigwedge A$ exist for arbitrary subsets $A$ of torsion classes. A subset $A \subseteq L$ is an antichain if the elements in $A$ are not comparable. The order ideal generated by $A$ is the set of $w \in L$ such that $w \leqslant a$ for some element $a \in A$.

Recall that the canonical join representation of an element $w$ is the unique "lowest" way to write $w$ as the join of smaller elements. Below, we make these notions precise. A join representation of an element $w$ in a complete lattice is an expression $w=\bigvee A$, where $A$ is a (possibly infinite) subset of $L$. We say that $\bigvee A$ is irredundant if $\bigvee A^{\prime}<$ $\bigvee A$, for each proper subset $A^{\prime} \subsetneq A$. Observe that if $\bigvee A$ is irredundant, then $A$ is an antichain.

Consider the set of all irredundant join representation for $w$. (Note that $\bigvee\{w\}$ is an irredundant join representation of $w$.) We partially order the irredundant join representations of $w$ as follows: Say $A \ll B$ if the order ideal generated by $A$ is contained in the order ideal generated by $\bar{B}$. Equivalently, $A \ll B$ if, for each $a \in A$, there exists some $b \in B$ such that $a \leqslant b$. Informally, we say that $A$ is "lower" than $B$. When $A$ is strictly "lower" than $B$ (e.g. $A \ll B$ and $A \neq B$ ) then we write $A \ll B$.

The canonical join representation of $w$ is the unique lowest irredundant join representation $\bigvee A$ of $w$, when such a representation exists. In this case, we also say that the set $A$ is a canonical join representation. The elements of $A$ are called canonical joinands of $w$. If $w$ is join-irreducible then $\bigvee\{w\}$ is the canonical join representation of $w$. Conversely, each canonical joinand is join-irreducible.

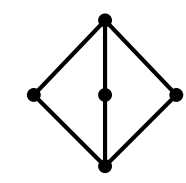

FiguRE 3. The top element does not have a canonical join representation.

REMARK 3.3. In general, the canonical join representation of an element may not exist. For example, see Figure 3. Observe that the join of each pair of atoms is a minimal, 
irredundant join representation of the top element. Since there is no unique such join representation, we conclude that the canonical join representation does not exist.

The canonical join complex of $L$, denoted $\Gamma(L)$, is the collection of subsets $A \subseteq L$ such that $A$ is a canonical join representation. In the following proposition (essentially [16, Proposition 2.2]) we show that $\Gamma(L)$ is closed under taking subsets. In the statement of [16, Proposition 2.2], the lattice $L$ is finite. A standard argument from lattice theory shows that the proposition also holds when $L$ is a complete meetsemilattice.

Proposition 3.4. Suppose that $\bigvee A$ is a canonical join representation in a complete meet-semilattice L. Then, for each $A^{\prime} \subseteq A$, the join $\bigvee A^{\prime}$ is also canonical join representation.

With Proposition 3.4, we conclude that the canonical join complex $\Gamma$ (tors $\Lambda$ ) is indeed a simplicial complex. We are finally prepared to prove Theorem 1.8. In the next proposition, we tackle the easier direction of the proof.

Proposition 3.5. Suppose that $\mathcal{E}$ is a collection of bricks over $\Lambda$. If the set $\{\mathscr{F}$ ilt $(\operatorname{Gen} M): M \in \mathcal{E}\}$ is a canonical join representation, then each pair of modules $M$ and $M^{\prime}$ is hom-orthogonal.

Proof. By Proposition 3.4, it is enough to show that the statement holds when $\mathcal{E}$ contains two elements, say $M$ and $M^{\prime}$. We write $\mathcal{T}$ for $\mathscr{F} i l t($ Gen $M) \vee \mathscr{F} i l t\left(\right.$ Gen $\left.M^{\prime}\right)$.

Suppose that $f: M^{\prime} \rightarrow M$ is a nonzero homomorphism, and write $N$ for $\operatorname{Im} f$. Since $\mathscr{F}$ ilt $($ Gen $M) \vee \mathscr{F}$ ilt $\left(\right.$ Gen $\left.M^{\prime}\right)$ is irredundant, it follows that $N$ is not isomorphic to $M$. Hence, $M / N$ is a proper factor of $M$, and the torsion class $\mathscr{F} i l t(\operatorname{Gen}(M / N))$ is strictly contained in $\mathscr{F} i l t(\operatorname{Gen} M)$. (Indeed, if $M$ belongs to $\mathscr{F} i l t(\operatorname{Gen}(M / N)$ ), then there exists a submodule $Y \subseteq M$ that is also a proper factor of $M$. That is a contradiction to the fact that $M$ is a brick.)

Finally, we observe that $\mathscr{F} i l t(\operatorname{Gen} M / N) \vee \mathscr{F} i l t\left(\operatorname{Gen} M^{\prime}\right)$ is an irredundant join representation for $\mathcal{T}$, and because $\mathscr{F} i l t(\operatorname{Gen} M / N) \subsetneq \mathscr{F} i l t(\operatorname{Gen} M)$,

$$
\left\{\mathscr{F} i l t(\operatorname{Gen} M / N), \mathscr{F} i l t\left(\operatorname{Gen} M^{\prime}\right)\right\} \ll\left\{\mathscr{F} i l t(\operatorname{Gen} M), \mathscr{F} i l t\left(\operatorname{Gen} M^{\prime}\right)\right\} \text {. }
$$

That is a contradiction to the fact that $\mathscr{F} i l t(\operatorname{Gen} M) \vee \mathscr{F} i l t\left(\right.$ Gen $\left.M^{\prime}\right)$ is the canonical join representation for $\mathcal{T}$.

Next, we consider the reverse implication of Theorem 1.8. As above, let $\mathcal{E}$ be a collection of hom-orthogonal bricks. We write $\mathcal{T}$ for the torsion class $\bigvee\{\mathscr{F} i l t(\operatorname{Gen} M)$ : $M \in \mathcal{E}\}$, and argue that $\bigvee\{\mathscr{F} i l t(\operatorname{Gen} M): M \in \mathcal{E}\}$ is the canonical join representation of $\mathcal{T}$. This will require the following lemma. For context, recall that a torsion class need not have any upper or lower cover relations. (For example, the torsion class $\mathcal{I}_{\infty}$ in the Kronecker quiver from Example 3.2 does not cover any other torsion class.)

Lemma 3.6. Suppose that $\mathcal{E}$ is a collection of hom-orthogonal bricks over $\Lambda$ and let $\mathcal{T}=\bigvee\{\mathscr{F} i l t(\operatorname{Gen} M): M \in \mathcal{E}\}$. Then for each module $M \in \mathcal{E}:$

(1) there is a torsion class $\mathcal{S} \lessdot \mathcal{T}$ such that $\mathcal{T}=\mathscr{F}$ ilt $(\mathcal{S} \cup M)$;

(2) $M$ is a minimal extending module for $\mathcal{S}$; and

(3) $\mathcal{S}$ contains $\mathcal{E} \backslash\{M\}$.

Proof. The torsion class $\mathcal{T}=\bigvee\{\mathscr{F} i l t(\operatorname{Gen}(M)): M \in \mathcal{E}\}$ is the smallest torsion class in tors $\Lambda$ that contains the modules in $\mathcal{E}$. Thus, a module $N$ belongs to $\mathcal{T}$ if and only if $N$ admits a filtration $N=N_{l} \supsetneq \cdots \supsetneq N_{0}=0$ such that each $N_{i} / N_{i-1}$ is a factor of some indecomposable module in $\mathcal{E}$. For each $M \in \mathcal{E}$, we claim that $M$ is a minimal co-extending module for the torsion-free class $\mathcal{T}^{\perp}$. 
First we check Property $\left(\mathrm{P}^{\prime}\right)$. Suppose that $Y$ is an indecomposable proper submodule of $M$ and that $Y \notin \mathcal{T}^{\perp}$. So, there exists a module $N \in \mathcal{T}$ and a nonzero homomorphism $f: N \rightarrow Y$. We may assume, without loss of generality, that $f$ is injective (if not, we take the map $f: N / \operatorname{ker} f \rightarrow Y$, noting that $N / \operatorname{ker} f$ is in $\mathcal{T}$ by closure under factors). From the filtration of $N$ described above, observe that the submodule $N_{1}$ is a factor of $M^{\prime}$, for some $M^{\prime} \in \mathcal{E}$. Also $f\left(N_{1}\right)$ is a non-trivial submodule of $Y$. So, we have the following sequence of homomorphisms

$$
M^{\prime} \rightarrow N_{1} \rightarrow f\left(N_{1}\right) \subseteq Y \subseteq M .
$$

The composition of these homomorphisms is non-zero, contradicting our hypothesis that $\operatorname{dim} \operatorname{Hom}_{\Lambda}\left(M^{\prime}, M\right)=0$. Therefore, $Y \in \mathcal{T}^{\perp}$. Futhermore, since $M \in \mathcal{T}$, Property $\left(\mathrm{P} 3^{\prime}\right)$ holds immediately.

To verify Property $\left(\mathrm{P} 2^{\prime}\right)$, suppose that $0 \rightarrow F \stackrel{i}{\rightarrow} X \stackrel{\pi}{\rightarrow} M \rightarrow 0$ is a non-split exact sequence with $F \in \mathcal{T}^{\perp}$, and that $X \notin \mathcal{T}^{\perp}$. As above, let $f: N \rightarrow X$ be a non-zero homomorphism, where $N \in \mathcal{T}$ indecomposable. We may again assume that $f$ is injective, and in particular the restriction of the map to $N_{1}$ is injective. As above, $N_{1}$ is a factor of some module $M^{\prime} \in \mathcal{E}$. Thus, we have the nonzero composite homomorphism:

$$
\tilde{f}: M^{\prime} \rightarrow N_{1} \rightarrow f\left(N_{1}\right) \subseteq X .
$$

Since $\operatorname{dim} \operatorname{Hom}_{\Lambda}\left(M^{\prime}, M\right)=0$ the composition $\pi \circ \tilde{f}$ is zero. Therefore, $\tilde{f}$ factors through the module $F$, and we have a nonzero homomorphism from $M^{\prime}$ to $F$. Since $F \in \mathcal{T}^{\perp}$, that is a contradiction. We conclude that $X \in \mathcal{T}^{\perp}$, and $M$ a minimal coextending module for $\mathcal{T}^{\perp}$. Proposition 2.12 implies that there exists a torsion class $\mathcal{S} \lessdot \mathcal{T}$ such that $\mathcal{T}=\mathscr{F} i l t(\mathcal{S} \cup\{M\})$ and $M$ a minimal extending module for $\mathcal{S}$.

To prove the third statement, suppose that $M^{\prime} \in \mathcal{E} \backslash\{M\}$ does not belong to $\mathcal{S}$. Proposition 2.6 implies that $M$ is a factor of $M^{\prime}$, and that is a contradiction. The statement follows.

The following proposition completes our proof of Theorem 1.8.

Proposition 3.7. Suppose that $\mathcal{E}$ is a collection of hom-orthogonal $\Lambda$ brick modules and write $\mathcal{T}$ for the torsion class $\bigvee\{\mathscr{F}$ ilt $(\operatorname{Gen} M): M \in \mathcal{E}\}$. Then the expression $\bigvee\{\mathscr{F}$ ilt $(\operatorname{Gen} M): M \in \mathcal{E}\}$ is the canonical join representation of $\mathcal{T}$.

Proof. We assume that $\mathcal{E}$ has at least two elements (otherwise the statement follows from Theorem 1.5). First we show that $\bigvee\{\mathscr{F} i l t(\operatorname{Gen} M): M \in \mathcal{E}\}$ is irredundant. Let $M \in \mathcal{E}$, and consider the torsion class $\mathcal{T}^{\prime}=\bigvee\left\{\mathscr{F}\right.$ ilt $\left(\right.$ Gen $\left.\left.M^{\prime}\right): M^{\prime} \in \mathcal{E} \backslash\{M\}\right\}$. By Lemma 3.6, there exists $\mathcal{S} \lessdot \mathcal{T}$ such that each module in $\mathcal{E} \backslash\{M\}$ lies in $\mathcal{S}$. Thus $\mathcal{T}^{\prime} \leqslant \mathcal{S} \lessdot \mathcal{T}$.

Next, we show the expression $\bigvee\{\mathscr{F} i l t(\operatorname{Gen} M): M \in \mathcal{E}\}$ is the unique lowest irredundant join representation for $\mathcal{T}$. Suppose that $\bigvee A$ is another irredundant join representation. We claim that for each torsion class $\mathscr{F} i l t(\operatorname{Gen} M)$ such that $M \in \mathcal{E}$, there exists some $\mathcal{G} \in A$ such that $\mathscr{F} i l t($ Gen $M) \leqslant \mathcal{G}$. (Thus, $\{\mathscr{F}$ ilt $($ Gen $M): M \in \mathcal{E}\}$ is "lower" than $A$.) Fix a module $M \in \mathcal{E}$, let $\mathcal{S}$ be the torsion class covered by $\mathcal{T}$ satisfying $\mathcal{T}=\mathscr{F} i l t(\mathcal{S} \cup\{M\})$ and $M$ is a minimal extending module for $\mathcal{S}$. (Such a torsion class $\mathcal{S}$ exists by Lemma 3.6.) Observe that there exists $\mathcal{G} \in A$ such that $\mathcal{G} \nsubseteq \mathcal{S}$. Indeed, if each $\mathcal{G}$ is contained in $\mathcal{S}$, then $\bigvee A \subseteq \mathcal{S}$. Let $N \in \mathcal{G} \backslash \mathcal{S}$. Proposition 2.6 implies that $M$ is a factor of $N$, hence $M \in \mathcal{G}$. We conclude that $\mathscr{F}$ ilt $(\operatorname{Gen} M) \leqslant \mathcal{G}$. We have proved the claim and the proposition.

REMARK 3.8. When tors $\Lambda$ is finite (equivalently, when $\Lambda$ is $\tau$-rigid finite) each joinirreducible torsion class is completely join-irreducible. Thus, the canonical join complex $\Gamma($ tors $\Lambda)$ is isomorphic to the complex of hom-orthogonal bricks. Moreover, the 
proof Proposition 3.7 implies that the number of canonical joinands of $\mathcal{T} \in$ tors $\Lambda$ is equal to the number of torsion classes $\mathcal{S}$ covered by $\mathcal{T}$. More precisely:

COROLlary 3.9. Suppose that $\mathcal{T}$ is a torsion class over $\Lambda$ with the following property: for every torsion class $\mathcal{S}$ with $\mathcal{S}<\mathcal{T}$, there is a torsion class $\mathcal{T}^{\prime}$ such that $\mathcal{S} \leqslant \mathcal{T}^{\prime} \lessdot \mathcal{T}$. Then the canonical join representation of $\mathcal{T}$ is equal to

$$
\bigvee\left\{\mathscr{F} i l t(\operatorname{Gen}(M)): M \text { is a minimal co-extending module of } \mathcal{T}^{\perp}\right\} \text {. }
$$

In particular, if $\Lambda$ is $\tau$-rigid finite, each torsion class has a canonical join representation.

Proof. The statement follows immediately form Corollary 2.9.

Indeed, the canonical join representation "sees" the geometry of the Hasse diagram for any finite lattice. We summarize this useful fact below (see [5, Proposition 2.2]).

Proposition 3.10. Suppose that $L$ is a finite lattice, and for each element $w \in L$ the canonical join representation of $w$ exists. Then, for each $w \in L$, the number of canonical joinands of $w$ is equal to the number of elements covered by $w$.
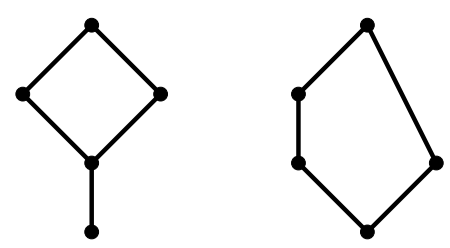

FiguRE 4. Two nonisomorphic lattices with isomorphic canonical join complexes.

REMARK 3.11. It is natural to ask if the canonical join complex $\Gamma$ (tors $\Lambda$ ) characterizes the underlying algebra $\Lambda$ or the torsion theory of $\Lambda$. In fact, non-isomorphic algebras may have the same torsion theory. We explore such an example in the following section when we show that the algebra $R A_{n}$ (an algebra of finite representation type for all $n$ ) has the same torsion theory as the preprojective algebra $\Pi A_{n}$ (which is not finite representation type for $n \geqslant 4$ ).

Furthermore, nonisomorphic lattices $L$ and $L^{\prime}$ may have isomorphic canonical join complexes. For example, consider the two (nonisomorphic) lattices shown in Figure 4. It is an easy exercise to check that canonical join complex of both lattices consists of an edge and an isolated vertex. (See Example 1.6.)

\section{4. tors $R A_{n}$ AND THE WEAK ORDER ON $A_{n}$}

Mizuno showed (in [15, Theorem 2.3]) that the lattice of torsion classes for the preprojective algebra of Dynkin type $W$ is isomorphic to the weak order on the associated Weyl group, when $W$ is simply laced. In this last section, we construct a different algebra, which we refer to as $R A_{n}$, and show that tors $R A_{n}$ is isomorphic to the weak order on $A_{n}$. This is carried out in two steps: First, in Theorem 4.5, we show that the canonical join complex of tors $R A_{n}$ is isomorphic to the canonical join complex of the weak order on $A_{n}$. Second, in Proposition 4.19, we map each cover relation $\mathcal{T} \lessdot \mathcal{T}^{\prime}$ in tors $R A_{n}$ bijectively to a cover relation of permutations in weak order. Before we begin, we establish some useful notation. Throughout we write $[n]$ for the set $\{1,2, \ldots, n\},[i, k]$ for the set $\{i, i+1, \ldots, k\}$ and $(i, k)$ for $\{i+1, \ldots, k-1\}$. 
4.1. The ALGeBRA $R A_{n}$. Let $Q$ be the quiver with vertex set $Q_{0}=[n]$ and arrows $Q_{1}=\left\{a_{i}: i \rightarrow i+1, a_{i}^{*}: i+1 \rightarrow i\right\}_{i \in[n-1]}$. Define $I$ to be the two-sided ideal in the path algebra $k Q$ generated by all two-cycles, $I=\left\langle a_{i} a_{i}^{*}, a_{i}^{*} a_{i} \mid i \in[n-1]\right\rangle$, and define $R A_{n}$ to be the algebra $k Q / I$.

Recall that a representation of $(Q, I)$ is a collection of vector spaces $M(x)$, one for each vertex $i \in Q_{0}$, and linear maps $M\left(a_{i}\right): M(i) \rightarrow M(i+1), M\left(a_{i}^{*}\right): M(i+1) \rightarrow$ $M(i)$, for each of the arrows in $Q$, that satisfy the relations given by $I$. We will make generous use of the equivalence between the category of modules over $R A_{n}$ and that of the representations of the bound quiver $(Q, I)$, generally referring to the objects of interest as modules, while describing them as representations. The support of a representation is the set of vertices $i$ for which $M(i) \neq 0$.

Proposition 4.1. There are finitely many isoclasses of indecomposable representations of $R A_{n}$ for each $n$.

Proof. The algebra $R A_{n}$ is gentle (see [3]) with no band modules since any cycle contains a 2-cycle, each of which lies in $I$. By the work of Butler-Ringel [7], then, there are finitely many isoclasses of indecomposable modules.

As a quiver representation, each indecomposable module $M$ (up to isomorphism) over $R A_{n}$ corresponds to a connected subquiver $Q_{M}$ of $Q$ satisfying the condition that at most one of either $a_{i}$ or $a_{i}^{*}$ belongs to $\left(Q_{M}\right)_{1}$. Thus, each indecomposable module can be identified with an orientation of a type-A Dynkin diagram with rank less than or equal to $n$. More precisely, the quiver representation corresponding to $Q_{M}$ satisfies:

- $M(i)=k$ for all $i \in\left(Q_{M}\right)_{0}$ and $M(i)=0$ for $i \notin\left(Q_{M}\right)_{0}$;

- $M(a) \neq 0$ if and only if $a \in\left(Q_{M}\right)_{1}$.

Proposition 4.2. Each indecomposable module over $R A_{n}$ is a brick. In particular, the canonical join complex of tors $R A_{n}$ is isomorphic to the complex of hom-orthogonal indecomposable modules over $R A_{n}$.

Proof. Let $M$ be an indecomposable module over $R A_{n}$ and let $Q_{M}$ be the corresponding quiver. An endomorphism $f: M \rightarrow M$ is a set of maps $f=\left(f_{i}\right)_{i \in Q_{1}}$ where $f_{i}: M(i) \rightarrow M(i)$ and for every arrow $a: i \rightarrow j \in Q_{1}$, the composition $M(a) \circ f_{i}$ is equal to $f_{j} \circ M(a)$. Since $M(i)=k$ for all $i \in\left(Q_{M}\right)_{0}$, the map $M(a): k \rightarrow k$ is just a scalar multiplication. Therefore $f_{i}=f_{j}$ for each $i, j \in\left(Q_{M}\right)_{0}$, and hence $f$ is a scalar multiple of the identity map.

From now on, we abuse notation and refer to $\Gamma$ (tors $\left.R A_{n}\right)$ as the complex of hom-orthogonal indecomposable modules over $R A_{n}$. (Although, more precisely, $\Gamma$ (tors $\left.R A_{n}\right)$ is a simplicial complex on the set of join-irreducible torsion classes $\mathscr{F}$ ilt $(\operatorname{Gen}(M))$ in tors $R A_{n}$, not the set of indecomposable modules.)

We close this section with a technical lemma that will be useful in Section 4.3.

Lemma 4.3. Suppose that $M$ is an indecomposable module over $R A_{n}$, and $S$ is an interval in $[n]$ containing $\operatorname{supp}(M)$. Then:

(1) $M$ is a submodule of some indecomposable $M^{\prime}$ with $\operatorname{supp}\left(M^{\prime}\right)=S$ and

(2) there is an indecomposable $M^{\prime \prime}$ with $\operatorname{supp}\left(M^{\prime \prime}\right)=S$, of which $M$ is a quotient.

Proof. We prove only the first statement, since the second is similar by Proposition 4.7. Let $Q_{M}$ be the quiver associated with $M$, and write $[p, q]=\operatorname{supp}(M)$. Let $Q_{M^{\prime}}$ be any quiver with support equal to $S$ satisfying the following: the orientation of $Q_{M^{\prime}}$ on the interval $[p, q]$ coincides with that of $Q_{M}$, and $Q_{M^{\prime}}$ contains the the arrows $a_{p-1}$ if $p-1 \in S$ and $a_{q}^{*}$ if $q+1 \in S$. Since $Q_{M}$ is a connected successor closed subquiver of $Q_{M^{\prime}}$, we obtain the desired result. 
4.2. NONCROSSING ARC DIAGRAMS AND CANONICAL JOIN REPRESENTATIONS. In this section, we construct a model for the canonical join complex of tors $R A_{n}$ called the noncrossing arc complex. The noncrossing arc complex was first defined in [16] where it was used to study certain aspects of the symmetric group. (We will make use of this connection in the following section.) Informally, the noncrossing arc complex is a simplicial complex whose faces are collections of non-intersecting curves called arcs. We will see that the "noncrossing" criteria that defines such a face also encodes the hom-orthogonality of indecomposable modules in $\bmod R A_{n}$.

A noncrossing arc diagram on $n+1$ nodes consists of a vertical column of nodes, labeled $0, \ldots, n$ in increasing order from bottom to top, together with a (possibly empty) collection of curves called arcs. Each arc $\alpha$ has two endpoints, and travels monotonically up from its bottom endpoint $b(\alpha)$ to its top endpoint $t(\alpha)$. For each node in between, $\alpha$ passes either to the left or to the right. Each pair of arcs $\alpha$ and $\beta$ in a diagram satisfies two compatibility conditions:

(C1) $\alpha$ and $\beta$ do not share a bottom endpoint or a top endpoint;

(C2) $\alpha$ and $\beta$ do not cross in their interiors.

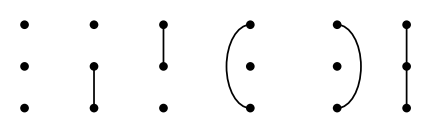

FiguRE 5. The noncrossing arc diagrams on 3 nodes.

Each arc is considered only up to combinatorial equivalence. That is, each arc $\alpha$ is characterized by its endpoints and which side each node the arc passes (either left or right) as it travels from $b(\alpha)$ up to $t(\alpha)$. Furthermore, a collection of arcs is drawn so as to have the smallest number of intersections. The support of an arc $\alpha$, written $\operatorname{supp}(\alpha)$, is the set $[b(\alpha), t(\alpha)]$. We write $\operatorname{supp}^{\circ}(\alpha)$ for the set $(b(\alpha), t(\alpha))$. We say that $\alpha$ has full support if $\operatorname{supp}(\alpha)=[0, n]$. We say that $\beta$ is a subarc of $\alpha$ if both of the following conditions are satisfied:

- $\operatorname{supp}(\beta) \subseteq \operatorname{supp}(\alpha)$;

- $\alpha$ and $\beta$ pass on the same side of each node in $\operatorname{supp}^{\circ}(\beta)$.

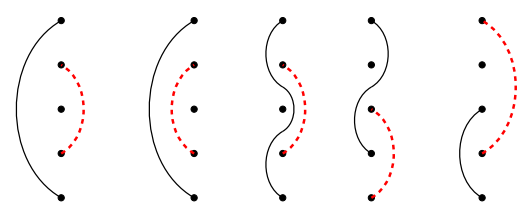

FiguRE 6. Some pairs of compatible arcs.

A set of arcs are compatible if there is a noncrossing arc diagram that contains them. We define the noncrossing arc complex on $n+1$ nodes to be the simplicial complex whose vertex set is the set of arcs and whose face set is the collection of all sets of compatible arcs. (We view each collection of compatible arcs as a noncrossing arc diagram. When we refer to "the set of arcs" we mean "the set of noncrossing arc diagrams, each of which contains precisely one arc".) The next proposition is a combination of [16, Proposition 3.2 and Corollary 3.6]. Recall that a simplicial complex is flag if its minimal non-faces have size equal to 2. Equivalently, a subset $F$ of vertices is a face if and only if each pair of vertices is a face. 
Proposition 4.4. A collection of arcs can be drawn together in a noncrossing arc diagram if and only if each pair of arcs is compatible. Thus, the noncrossing arc complex is flag.

Our goal is to prove:

THEOREM 4.5. The canonical join complex of the lattice tors $R A_{n}$ is isomorphic to the noncrossing arc complex on $n+1$ nodes.

We begin the proof of Theorem 4.5 by mapping vertices to vertices. More precisely, we define a bijection $\sigma$ from the set of indecomposable modules over $R A_{n}$ to the set of arcs on $n+1$ nodes.

For an arc $\alpha$ with support $[p-1, q]$ we define:

$$
\begin{aligned}
& R(\alpha)=\{i \in[p, q-1]: \alpha \text { passes on the right side of } i\} ; \\
& L(\alpha)=\{i \in[p, q-1]: \alpha \text { passes on the left side of } i\} .
\end{aligned}
$$

For an indecomposable $R A_{n}$ module with support $[p, q] \subseteq[n]$, we define:

$$
\begin{aligned}
R(M) & =\left\{i \in[p, q-1]: a_{i} \text { acts nontrivially on } M\right\} \\
L(M) & =\left\{i \in[p, q-1]: a_{i}^{*} \text { acts nontrivially on } M\right\} .
\end{aligned}
$$

Just as an arc is determined by its endpoints and the binary Left-Right data, so too is an indecomposable module over $R A_{n}$ determined by the binary data of the action of its Lowering-Raising arrows ( $a_{i}^{*}$ and $a_{i}$, respectively). Therefore, we have the following:

Proposition 4.6. Let $\sigma$ be the map which sends an indecomposable $R A_{n}$ module $M$ with support $[p, q-1]$ to the arc $\sigma(M)=\alpha$ satisfying

- $b(\alpha)=p-1$ and $t(\alpha)=q$;

- $L(\alpha)=L(M)$;

- $R(\alpha)=R(M)$.

Then $\sigma$ is a bijection from the set of indecomposable modules over $R A_{n}$ the set of arcs on $n+1$ nodes. (See Figure 7.)

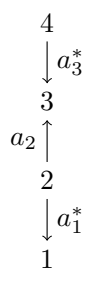

(A) $Q_{M}$ for $R A_{4}$

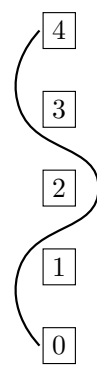

(B) The $\operatorname{arc} \sigma(M)$

FiguRE 7. Visualization of $\sigma(M)$ and $Q_{M}$ for a module over $R A_{4}$.

For the remainder of the section, we let $M$ and $M^{\prime}$ be indecomposable modules, and write $\alpha$ for $\sigma(M)$ and $\alpha^{\prime}$ for $\sigma\left(M^{\prime}\right)$. We wish is to reinterpret the hom-orthogonality of $M$ and $M^{\prime}$ in terms of certain subarcs of $\alpha$ and $\alpha^{\prime}$. Recall that a quiver $Q^{\prime}$ is called a predecessor closed subquiver of $Q$ if $i \rightarrow j$ with $j \in Q^{\prime}$ implies $i \in Q^{\prime}$. Successor closed subquivers are defined similarly. The following result is well-known (and an easy exercise). See $[9$, Section 2]. 
Proposition 4.7. Suppose that $M$ and $M^{\prime}$ are indecomposable modules over $R A_{n}$ and let $Q_{M}$ and $Q_{M^{\prime}}$ be the corresponding quivers. Then:

(1) $M^{\prime}$ is a quotient of $M$ if and only if $Q_{M^{\prime}}$ is a connected predecessor closed subquiver of $Q_{M}$.

(2) $M^{\prime}$ is a submodule of $M$ if and only if $Q_{M^{\prime}}$ is a connected successor closed subquiver of $Q_{M}$.

We define an analogous notion for arcs. We say that $\beta$ is a predecessor closed subarc of $\alpha$ if $\beta$ is a subarc of $\alpha$, and $\alpha$ does not pass to the right of $b(\beta)$, nor to the left of $t(\beta)$. Similarly, $\beta$ is a successor closed subarc if $\alpha$ does not pass to the left of $b(\beta)$ nor to the right of $t(\beta)$. Observe that each predecessor closed subarc of $\alpha$ corresponds (via the map $\sigma$ ) to an indecomposable quotient of $M$-that is, a predecessor closed subquiver of $Q_{M}$. (The analogous statement holds for successor closed subarcs of $\alpha$.) The next result is from Crawley-Boevey [9, Section 2], rephrased for our context.

Proposition 4.8. Let $\alpha$ and $\alpha^{\prime}$ be arcs on $n+1$ nodes, and write $M$ and $M^{\prime}$ for the corresponding indecomposable modules over $R A_{n}$. Then the vector space $\operatorname{Hom}_{R A_{n}}\left(M, M^{\prime}\right)$ has dimension equal to the number of predecessor closed subarcs of $\alpha$ which are also successor closed subarcs of $\alpha^{\prime}$.

We argue that $\alpha$ and $\alpha^{\prime}$ are compatible if and only if there exists no $\operatorname{arc} \beta$ that is a predecessor closed subarc of one which is simultaneously a successor closed subarc of the other.

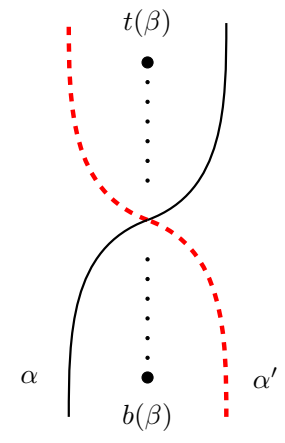

FiguRE 8. Suppose that $\beta$ is a predecessor closed subarc of $\alpha$ and a successor closed subarc of $\alpha^{\prime}$. Then the endpoints of $\beta$ lie between these two arcs. The two arcs switch from left side to right side (and vice versa) as they travel from $b(\beta)$ to $t(\beta)$.

The following two lemmas give one direction of that argument. Figure 8 may help build some intuition.

At times it will be convenient to consider the relative position of a pair of "overlapping" arcs.

We say that $\alpha$ and $\alpha^{\prime}$ overlap if the set

$$
\left(\operatorname{supp}(\alpha) \cap \operatorname{supp}^{\circ}\left(\alpha^{\prime}\right)\right) \cup\left(\operatorname{supp}\left(\alpha^{\prime}\right) \cap \operatorname{supp}^{\circ}(\alpha)\right)
$$

is nonempty. For two $\operatorname{arcs} \alpha$ and $\alpha^{\prime}$ that overlap, we say that $\alpha^{\prime}$ is right of $\alpha$ if both of the following hold:

- $\left(L\left(\alpha^{\prime}\right) \cup\left\{t\left(\alpha^{\prime}\right), b\left(\alpha^{\prime}\right)\right\}\right) \cap(\operatorname{supp}(\alpha)) \subseteq L(\alpha)$

- $\{t(\alpha), b(\alpha)\} \cap\left(\operatorname{supp}\left(\alpha^{\prime}\right)\right) \subseteq R\left(\alpha^{\prime}\right)$.

For example, in Figure 6, the dashed arc is right of the solid arc. Observe that if $\alpha^{\prime}$ is right of $\alpha$ then the two arcs are compatible. 
LEMMA 4.9. Suppose that $\alpha$ and $\alpha^{\prime}$ are distinct arcs that share a bottom endpoint or a top endpoint. Then there exists an arc $\beta$ satisfying: $\beta$ is a predecessor closed subarc of one arc, either $\alpha$ or $\alpha^{\prime}$, and a successor closed subarc of the other arc.

Proof. By symmetry, we assume that $\alpha$ and $\alpha^{\prime}$ share a bottom node, and, without loss of generality, this bottom endpoint is equal to 0 . Let $q$ be the smallest number such that either of two conditions below is satisfied:

- $\alpha$ and $\alpha^{\prime}$ pass on opposite sides of $q$;

- $q=\min \left\{t(\alpha), t\left(\alpha^{\prime}\right)\right\}$.

Let $\beta$ be the arc with endpoints $b(\beta)=0$ and $t(\beta)=q$ such that $\beta$ is a subarc of $\alpha$. (Note, if $q=t(\alpha)$ then $\beta$ coincides with $\alpha$. If $q \neq t(\alpha)$, we can visualize $\beta$ by cutting $\alpha$ where it passes beside the node $q$, and anchoring the resulting segment at $q$.) Since $\alpha$ and $\alpha^{\prime}$ pass on the same side of each node in the set $[0, q-1]$, we conclude that $\beta$ is also a subarc of $\alpha^{\prime}$.

At least one of the two arcs, $\alpha$ or $\alpha^{\prime}$, passes $q$. By symmetry, assume that this is the arc $\alpha$, and assume that $\alpha$ passes on the right side of $q$. Then $\beta$ is a predecessor closed subarc of $\alpha$ and also a successor closed subarc of $\alpha^{\prime}$.

Lemma 4.10. Suppose that $\alpha$ and $\alpha^{\prime}$ are distinct arcs that neither share a bottom nor top endpoint. If $\alpha$ and $\alpha^{\prime}$ intersect in their interiors, then there exists an arc $\beta$ which is a predecessor closed subarc of one arc and a successor closed subarc of the other.

Proof. First, we consider the case in which one of the two arcs is a subarc of the other. By symmetry, we assume that $\alpha^{\prime}$ is a subarc of $\alpha$. Observe that $b\left(\alpha^{\prime}\right)$ and $t\left(\alpha^{\prime}\right)$ must be on opposite sides of $\alpha$. (Otherwise, $\alpha$ and $\alpha^{\prime}$ can be drawn so they do not cross.) Therefore, by definition, either $\alpha^{\prime}$ is a predecessor closed or successor closed subarc of $\alpha$.

Next, suppose that $\alpha^{\prime}$ and $\alpha$ pass on the same side of each node in the set $\operatorname{supp}^{\circ}(\alpha) \cap \operatorname{supp}^{\circ}\left(\alpha^{\prime}\right)$, but neither arc is a subarc of the other. By symmetry, assume that $b\left(\alpha^{\prime}\right)<b(\alpha)<t\left(\alpha^{\prime}\right)<t(\alpha)$ and that $\alpha^{\prime}$ passes to the right of $b(\alpha)$. Observe that $\alpha$ also passes to the right of $t\left(\alpha^{\prime}\right)$. Otherwise, the arcs can be drawn so that $\alpha^{\prime}$ lies strictly to the right of $\alpha$, and the two arcs never cross. See Figure 9.

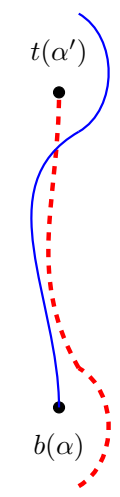

Figure 9. A demonstration of the proof of Lemma 4.10. The arc $\alpha$ is shown in blue, and $\alpha^{\prime}$ is shown in dashed red.

Let $\beta$ be the subarc of $\alpha$ with bottom endpoint $b(\alpha)$ and top endpoint $t\left(\alpha^{\prime}\right)$. Since $\alpha$ and $\alpha^{\prime}$ pass on the same side of each node where they overlap, $\beta$ is also a subarc of $\alpha^{\prime}$. We conclude that $\beta$ is a predecessor closed subarc of $\alpha$ that is also a successor closed subarc of $\alpha^{\prime}$. 
Finally, we assume that $\alpha$ and $\alpha^{\prime}$ pass on opposite sides of some node $p$ belonging to $\operatorname{supp}^{\circ}(\alpha) \cap \operatorname{supp}^{\circ}\left(\alpha^{\prime}\right)$. By symmetry, assume that $\alpha$ passes to the left side of $p$ and $\alpha^{\prime}$ passes to the right. Consider the set

$$
O=\left(\operatorname{supp}(\alpha) \cap \operatorname{supp}^{\circ}\left(\alpha^{\prime}\right)\right) \cup\left(\operatorname{supp}\left(\alpha^{\prime}\right) \cap \operatorname{supp}^{\circ}(\alpha)\right) .
$$

By way of contradiction, suppose for each $q \in O$, either $\alpha$ passes on the left side of $q$ or $\alpha^{\prime}$ passes on the right of $q$. Then for each $q \in O$, either $\alpha$ and $\alpha^{\prime}$ pass on the same side of $q$, or $q$ lies between them, with $\alpha$ on the left and $\alpha^{\prime}$ on the right. We conclude that $\alpha^{\prime}$ is strictly to the right of $\alpha$, and the two arcs never cross, a contradiction.

Thus, there exists some $q \in O$ satisfying: $\alpha$ does not pass to the left of $q$ nor does $\alpha^{\prime}$ pass to the right. We choose $p$ and $q$ so that $|p-q|$ is minimal. By symmetry, assume that $p<q$. Let $\beta$ be the subarc of $\alpha$ with endpoints $p<q$. The minimality of $|p-q|$ implies that $\beta$ is also a subarc of $\alpha^{\prime}$. We conclude that $\beta$ is a predecessor closed subarc of $\alpha$ that is also a successor closed subarc of $\alpha^{\prime}$.

Together, the previous two lemmas (and Proposition 4.8) imply that if $\alpha$ and $\alpha^{\prime}$ are not compatible, then there exists some homomorphism between $M$ and $M^{\prime}$. The next lemma completes the proof of Theorem 4.5.

Lemma 4.11. Suppose that $\alpha$ and $\alpha^{\prime}$ are compatible. Then there exists no arc $\beta$ that is predecessor closed subarc of one arc and a successor closed subarc of the other. Thus, the modules $M$ and $M^{\prime}$ corresponding to $\alpha$ and $\alpha^{\prime}$ under the bijection $\sigma$ are hom-orthogonal.

Proof. By way of contradiction, assume there exists an $\operatorname{arc} \beta$ that is both a predecessor closed subarc of $\alpha$ and a successor closed subarc of $\alpha^{\prime}$. Because $\alpha$ and $\alpha^{\prime}$ do not share a bottom endpoint, either $b(\alpha) \neq b(\beta)$ or $b\left(\alpha^{\prime}\right) \neq b(\beta)$. By symmetry, assume that $b(\alpha) \neq b(\beta)$. Thus, $\alpha$ passes strictly to the left of $b(\beta)$. Since $\alpha^{\prime}$ does not pass to the left of $b(\beta)$, we conclude that $\alpha^{\prime}$ lies strictly on the right side of $\alpha$, wherever the two arcs overlap.

For the moment, assume that $t(\beta) \in \operatorname{supp}^{\circ}(\alpha) \cap \operatorname{supp}^{\circ}\left(\alpha^{\prime}\right)$. On the one hand, $\alpha$ passes strictly to the right of $t(\beta)$. On the other hand, $\alpha^{\prime}$ passes strictly to the left of $t(\beta)$, so that $\alpha$ and $\alpha^{\prime}$ intersect in their interiors. (See Figure 8.) We obtain a similar contradiction if $t(\beta)=t(\alpha)$ or $t(\beta)=t\left(\alpha^{\prime}\right)$.

4.3. THE WEAK ORDER ON $A_{n}$ AND tors $R A_{n}$. In this section we prove that tors $R A_{n}$ is isomorphic to the weak order on $A_{n}$. We begin by reviewing the weak order and its connection to noncrossing arc diagrams.

Recall that the type-A Weyl group of rank $n$ is isomorphic to the symmetric group on $[n+1]$. It will be convenient for us to realize $A_{n}$ as the symmetric group on $\{0, \ldots, n\}=[0, n]$. For the remainder of the paper, we do not distinguish between the elements of $A_{n}$ and the permutations on $[0, n]$. We write $w \in A_{n}$ in its one-line notation as $w=w_{0} \ldots w_{n}$ where $w_{i}=w(i)$. An inversion of $w$ is a pair $\left(w_{i}, w_{j}\right)$ with $w_{i}>w_{j}$ and $i<j$. Each permutation is uniquely determined by its inversion set.

In the weak order, permutations are partially ordered by containment of their inversion sets. In particular, $w>v$ if and only if $\operatorname{inv}(v) \subseteq \operatorname{inv}(w)$ and $\operatorname{inv}(w) \backslash \operatorname{inv}(v)$ has precisely one element. This unique inversion is a descent for $w$. (Recall that a descent is an inversion $(q, p)$ such that $q=w_{i}$ and $p=w_{i+1}$.) In this way, each descent of $w$ corresponds bijectively to a permutation that is covered by $w$. It follows that $w$ is join-irreducible if and only if it has precisely one descent.

We describe a bijection $\delta$ from the set of permutations in $A_{n}$ to the set of noncrossing arc diagrams on $n+1$ nodes. For each descent $\left(w_{i}, w_{i+1}\right)$ of a permutation $w$, construct an arc $\alpha$ satisfying: $t(\alpha)=w_{i}, b(\alpha)=w_{i+1}$, and $w_{j} \in R(\alpha)$ (resp. 
$\left.w_{j} \in L(\alpha)\right)$ whenever $w_{j}$ is in the interval $(b(\alpha), t(\alpha))$ and $j>i+1$ (resp. $j<i$ ). The arc diagram $\delta(w)$ is then the union of these arcs. Since descents become arcs, it follows that $\delta$ restricts to a bijection from the set of join-irreducible permutations (on $[0, n])$ to the set of $\operatorname{arcs}$ (on $n+1$ nodes).
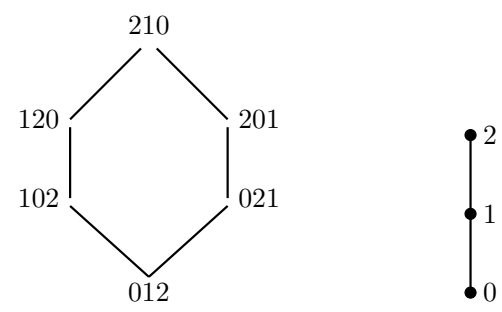

Figure 10. The weak order on the symmetric group $A_{2}$ and the noncrossing arc diagram corresponding to 210.

EXAMPLE 4.12. Consider the permutation $w=210$, the top element in the weak order on $A_{2}$. The noncrossing arc diagram $\delta(w)$ consists of two arcs, one connecting 0 to 1 , and the second connecting 1 to 2. (See Figure 10.) Each arc corresponds (via $\delta$ ) to a join-irreducible permutation. In this example, the arc connecting 0 to 1 corresponds to 102 , and the arc which connects 1 to 2 corresponds to 021 . Observe that that $210=\bigvee\{102,021\}$. In fact, $\bigvee\{102,021\}$ is the canonical join representation of 210 . This fact is no coincidence.

In general, the weak order on $A_{n}$ is a lattice in which each permutation has a canonical join representation [8]. The next theorem is a combination of [16, Theorem 3.1 and Corollary 3.4].

THEOREM 4.13. The bijection $\delta$ induces an isomorphism from the canonical join complex of the weak order on $A_{n}$ to the noncrossing arc complex on $n+1$ nodes.

We immediately obtain the following corollary.

COROLlary 4.14. The canonical join complex of $R A_{n}$ is isomorphic to the canonical join complex of the weak order on $A_{n}$.

Let us a consider the following composition:

$$
\Gamma\left(\text { tors } R A_{n}\right) \stackrel{\sigma}{\rightarrow}\{\text { Noncrossing arc diagrams on } n+1 \text { nodes }\} \stackrel{\delta^{-1}}{\longrightarrow} \Gamma\left(A_{n}\right) .
$$

Recall that the map $\sigma$ sends a collection of hom-orthogonal modules $\mathcal{E}$ to a collection of compatible $\operatorname{arcs} \sigma(\mathcal{E})=A$. By Theorem 4.13, $\delta^{-1}$ sends this collection of compatible arcs to the permutation $w=\bigvee\left\{\delta^{-1}(\alpha): \alpha \in A\right\}$, where this join $\bigvee\left\{\delta^{-1}(\alpha): \alpha \in A\right\}$ is the canonical join representation of $w$. Define a map $\phi: \operatorname{tors} R A_{n} \rightarrow A_{n}$ as follows:

$$
\phi: \bigvee\{\mathscr{F} \text { ilt }(\operatorname{Gen}(M)): M \in \mathcal{E}\} \mapsto \bigvee\left\{\delta^{-1}(\sigma(M)): M \in \mathcal{E}\right\} .
$$

Because each torsion class in tors $R A_{n}$ and each permutation in $A_{n}$ has a canonical join representation (see Corollary 3.9), and because the canonical join representation is unique, we conclude that $\phi$ is a bijection.

EXAmple 4.15. Consider the join of torsion classes $\mathcal{T}=\bigvee\{\{1\},\{2\}\}$ in tors $R A_{2}$. (We write $\{i\}$ for the torsion class consisting of the simple module at vertex $i$.) Observe that $\mathcal{T}$ is equal to the entire module category over $R A_{2}$. Since the simple modules 1 and 2 are hom-orthogonal, the join $\bigvee\{\{1\},\{2\}\}$ is the canonical join representation of $\mathcal{T}$. 
The map $\sigma$ sends $\{i\}$ to the arc with endpoints $i-1$ and $i$. Thus, $\sigma(\mathcal{T})$ is the noncrossing arc diagram with two arcs: one arc that connects 0 to 1 ; and another arc that connects 1 to 2 . Recall from Example 4.12 that the permutation associated to this diagram is 210 . Thus, $\phi(\mathcal{T})=210$.

Our goal is to show that $\phi$ is actually a lattice isomorphism. To that end, we now give an alternative description of $\phi$ in terms of inversion sets. Recall that permutations in $A_{n}$ are ordered by containment of inversion sets. Each inversion set $I=\operatorname{inv}(w)$ is transitively closed, meaning that whenever $\{(p, q),(q, r)\}$ is a subset of $I$ then $(p, r) \in I$. Consider the set $S$ of all pairs $(p, q)$ such that $p<q$ and $p, q \in[0, n]$, and write $\Omega$ for the power set of $S$. The transitive closure of $I$ is the unique smallest (under containment) set $\operatorname{tran}(I)$ in $\Omega$ that is transitively closed and contains $I$. Just as we compute the join of a set of torsion classes by taking their filtration closure, we compute the join of a set of permutations by taking their transitive closure. More precisely:

Proposition 4.16. Suppose that $U$ is a collection of permutations in $A_{n}$. The inversion set of the permutation $w=\bigvee U$ is equal to the transitive closure of the set $I=\{\operatorname{inv}(u): u \in U\}$.

Building on this analogy, let us define an "inversion set" for a torsion class of $\bmod R A_{n}$. For each indecomposable module $M$ with support $[p, r]$ we associate to $M$ the pair $\operatorname{inv}(M)=(p-1, r)$. We define the inversion set of a torsion class $\mathcal{T}$ to be the set of all pairs $\operatorname{inv}(M)$ such that $M \in \operatorname{ind}(\mathcal{T})$.

Lemma 4.17. Suppose that $S$ is a collection of indecomposable modules over $R A_{n}$. Then $\operatorname{inv}(\mathscr{F} i l t(S))$ is equal to the transitive closure of $\operatorname{inv}(S)$.

Proof. First, suppose that $(p-1, r) \in \operatorname{inv}(\mathscr{F} i l t(S))$, and let $M$ be an indecomposable module in $\mathscr{F}_{i l t}(S)$ with $\operatorname{supp}(M)=[p, r]$. Without loss of generality, we assume that $M$ is not simple. Thus, $p<r$. We prove that $(p-1, r) \in \operatorname{tran}(\operatorname{inv}(S))$ by induction on $l$.

Let $M=M_{l} \supsetneq M_{l-1} \supsetneq \cdots \supsetneq M_{0}=0$ be an $S$-filtration of $M$, and consider the short exact sequence:

$$
0 \rightarrow M_{l-1} \rightarrow M \rightarrow M / M_{l-1} \rightarrow 0 .
$$

Since $\operatorname{dim}\left(M_{l-1}(i)\right)+\operatorname{dim}\left(M / M_{l-1}(i)\right)=\operatorname{dim}(M(i))$ for each vertex $i \in Q_{0}$, we conclude that $\operatorname{supp}\left(M_{l-1}\right)=[p, q-1]$ and $\operatorname{supp}\left(M / M_{l-1}\right)=[q, r]$, for some $q \in(p, r)$. Because $M_{l-1}$ belongs to $\mathscr{F} i l t^{l-1}(S)$, our inductive hypothesis implies that $(p-1, q-1) \in$ $\operatorname{tran}(\operatorname{inv}(S))$. Also $M / M_{l-1} \in S$, so we have $(q-1, r) \in \operatorname{inv}(S)$. Thus, $(p-1, r) \in$ $\operatorname{tran}(\operatorname{inv}(S))$. The other containment follows immediately from Lemma 4.3.

Proposition 4.18. Suppose that $\mathcal{T}$ is a torsion class in tors $R A_{n}$. Then inversion set of $\mathcal{T}$ is equal to $\operatorname{inv}(\phi(\mathcal{T}))$.

Proof. First suppose that $\mathcal{T}$ is join-irreducible, so that $\mathcal{T}=\mathscr{F} i l t(\operatorname{Gen}(M))$, where $M$ is an indecomposable module over $R A_{n}$. Without loss of generality, we assume that $M$ has full support. As in Proposition 4.6, let $\alpha=\sigma(M)$, where $\alpha$ is defined by the condition that $L(\alpha)=L(M)$ and $R(\alpha)=R(M)$. (Recall from Section 4.2 that $L(M)$ is the set of indices of the arrows of type $a_{i}^{*}$ in $Q_{M}$ while $L(\alpha)$ is the set of nodes $i$ such that $\alpha$ passes on the left side of $i$.) Write $w$ for the join-irreducible permutation $\delta^{-1}(\alpha)$. On the one hand, a pair $(p-1, r)$ is an inversion of $w$ if and only if $p-1 \in L(\alpha) \cup\{0\}$ and $r \in R(\alpha) \cup\{n\}$. On the other hand, $(p-1, r)$ is $\operatorname{inv}(N)$ for some factor $N$ of $M$ if and only if $p-1 \in L(M) \cup\{0\}$ and $r \in R(M) \cup\{n\}$. (Equivalently, each factor of $M$ has support $[p, r]$ such that $p-1 \in L(M) \cup\{0\}$ and $r \in R(M) \cup\{n\}$.) We conclude that $\operatorname{inv}(\operatorname{Gen}(M))$ is equal to $\operatorname{inv}(\phi(\mathcal{T}))$. Since the 
inversion set of the permutation $\phi(\mathcal{T})$ is already transitively closed, we conclude that $\operatorname{inv}(\mathcal{T})$ is equal to $\operatorname{inv}(\phi(\mathcal{T}))$.

Now assume that $\mathcal{T}$ is not join-irreducible. Write $\bigvee\{\mathscr{F} i l t(\operatorname{Gen}(M)): M \in \mathcal{E}\}$ for its canonical join representation, and write $A$ for the set $\delta^{-1}(\sigma(\mathcal{E}))$. Recall that $\phi(\mathcal{T})=\bigvee\{w: w \in A\}$, and this join is computed by taking the transitive closure of the set $\{\operatorname{inv}(w): w \in A\}$. The join $\bigvee\{\mathscr{F} i l t(\operatorname{Gen}(M)): M \in \mathcal{E}\}$ is computed by taking the filtration closure of the set $\{\operatorname{Gen}(M): M \in \mathcal{E}\}$. By Lemma 4.17, $\operatorname{inv}(\mathcal{T})$ is equal to $\operatorname{inv}(\phi(\mathcal{T}))$.

We are now prepared to prove the main result of this section.

THEOREM 4.19. Let $\mathcal{T}^{\prime}$ and $\mathcal{T}$ be torsion classes in tors $R A_{n}$. Suppose that $\mathcal{T}^{\prime}$ is equal to $\mathscr{F}$ ilt $(\mathcal{T} \cup\{M\})$, where $M$ is a minimal extending module for $\mathcal{T}$. Then

$$
\operatorname{inv}\left(\mathcal{T}^{\prime}\right) \backslash \operatorname{inv}(\mathcal{T})=\{\operatorname{inv}(M)\}
$$

In particular, tors $R A_{n}$ is isomorphic to the weak order on $A_{n}$.

Proof. Write $(q, r)$ for the inversion $\operatorname{inv}(M)$. Proposition 4.17 says that $\operatorname{inv}\left(\mathcal{T}^{\prime}\right)=$ $\operatorname{tran}(\operatorname{inv}(\mathcal{T}) \cup\{(q, r)\})$. If $(q, r) \in \operatorname{inv}(\mathcal{T})$ then $\operatorname{inv}(\mathcal{T})$ is equal to $\operatorname{inv}\left(\mathcal{T}^{\prime}\right)$, and this contradicts the fact that $\phi$ is a bijection (because distinct permutations have distinct inversion sets). We claim that the $\operatorname{set} \operatorname{inv}(\mathcal{T} \cup\{(q, r)\})$ is transitively closed.

To prove this claim, first suppose that $p<q$ and $(p, q) \in \operatorname{inv}(\mathcal{T})$. We need to show that $(q, r) \in \operatorname{inv}(\mathcal{T})$. Let $M^{\prime}$ be an indecomposable module in $\mathcal{T}$ with $\operatorname{supp}(M)=$ $[p+1, q]$. Then, Lemma 4.3 implies that there exists a short exact sequence:

$$
0 \rightarrow M \rightarrow M^{\prime \prime} \rightarrow M^{\prime} \rightarrow 0,
$$

where $\operatorname{supp}\left(M^{\prime \prime}\right)=[p+1, r]$. Since $M$ is minimally extending, (P2) implies that $M^{\prime \prime} \in \mathcal{T}^{\prime}$. The statement follows. A similar argument shows that if there is some $s>r$ such that $(r, s) \in \operatorname{inv}(\mathcal{T})$ then $(q, s)$ also belongs to $\operatorname{inv}(\mathcal{T})$. We conclude that $\operatorname{inv}\left(\mathcal{T}^{\prime}\right)=\operatorname{inv}(\mathcal{T}) \cup\{(q, r)\}$, as desired. In particular, $\phi\left(\mathcal{T}^{\prime}\right)>\phi(\mathcal{T})$.

For any finite lattice, define $\operatorname{cov}_{\downarrow}(L)$ to be the set of pairs $\left(w^{\prime}, w\right)$ such that $w^{\prime}>w$. As is the case for tors $R A_{n}$ and $A_{n}$, suppose that each element $w^{\prime} \in L$ has a canonical join representation. Recall that the number of canonical joinands of $w^{\prime}$ is equal to the number of elements covered by $w^{\prime}$. (This is Proposition 3.10.) Thus, the number of pairs $\left(w^{\prime}, w\right)$ in $\operatorname{cov}_{\downarrow}(L)$ is equal to a weighted sum of the faces in $\Gamma(L)$, where each face is weighted by its size. In particular, the sets $\operatorname{cov}_{\downarrow}\left(\operatorname{tors} R A_{n}\right)$ and $\operatorname{cov}_{\downarrow}\left(A_{n}\right)$ are equinumerous. Since $\phi$ is a bijection, we conclude that the mapping $\left(\mathcal{T}^{\prime}, \mathcal{T}\right) \mapsto\left(\phi(\mathcal{T}), \phi\left(\mathcal{T}^{\prime}\right)\right)$ is a bijection from $\operatorname{cov}_{\downarrow}\left(\operatorname{tors} R A_{n}\right)$ to $\operatorname{cov}_{\downarrow}\left(A_{n}\right)$. Hence, the lattice tors $R A_{n}$ is isomorphic to the weak order on $A_{n}$.

Acknowledgements. The authors thank David Speyer and Gordana Todorov, who helped start this project. We also thank Alexander Garver and Thomas McConville, who led us to the correct statement of Theorem 1.8.

\section{REFERENCES}

[1] Takahide Adachi, Osamu Iyama, and Idun Reiten, $\tau$-tilting theory, Compos. Math. 150 (2014), no. $3,415-452$

[2] Sota Asai, Semibricks, to appear in Int. Math. Res. Not, https://arxiv.org/abs/1610.05860, 2018.

[3] Ibrahim Assem and Andrzej Skowroński, Iterated tilted algebras of type $\tilde{\mathbf{A}}_{n}$, Math. Z. 195 (1987), no. 2, 269-290.

[4] Maurice Auslander, Idun Reiten, and Sverre O. Smalø, Representation theory of Artin algebras, Cambridge Studies in Advanced Mathematics, vol. 36, Cambridge University Press, Cambridge, 1997, Corrected reprint of the 1995 original. 
[5] Emily Barnard, The canonical join complex, Electronic J. Combinatorics 26 (2019), no. 1, P1.24 (25 pages).

[6] Thomas Brüstle, David Smith, and Hipolito Treffinger, Wall and chamber structure for finitedimensional algebras, Preprint available: https://arxiv.org/abs/1805.01880, 2018.

[7] Michael C. R. Butler and Claus Michael Ringel, Auslander-Reiten sequences with few middle terms and applications to string algebras, Comm. Algebra 15 (1987), no. 1-2, 145-179.

[8] Nathalie Caspard, Claude Le Conte de Poly-Barbut, and Michel Morvan, Cayley lattices of finite Coxeter groups are bounded, Adv. in Appl. Math. 33 (2004), no. 1, 71-94.

[9] William Crawley-Boevey, Maps between representations of zero-relation algebras, J. Algebra 126 (1989), no. 2, 259-263.

[10] Laurent Demonet, Osamu Iyama, and Gustavo Jasso, $\tau$-tilting finite algebras, bricks, and $g$ vectors, Int. Math. Res. Not. IMRN (2019), no. 3, 852-892.

[11] Laurent Demonet, Osamu Iyama, Nathan Reading, Idun Reiten, and Hugh Thomas, Lattice theory of torsion classes, Preprint available: https://arxiv.org/abs/1711.01785, 2018.

[12] Alexander Garver and Thomas McConville, Lattice properties of oriented exchange graphs and torsion classes, Algebr. Represent. Theory 22 (2019), no. 1, 43-78.

[13] Osamu Iyama, Nathan Reading, Idun Reiten, and Hugh Thomas, Lattice structure of Weyl groups via representation theory of preprojective algebras, Compos. Math. 154 (2018), no. 6, $1269-1305$.

[14] Osamu Iyama, Idun Reiten, Hugh Thomas, and Gordana Todorov, Lattice structure of torsion classes for path algebras, Bull. Lond. Math. Soc. 47 (2015), no. 4, 639-650.

[15] Yuya Mizuno, Classifying $\tau$-tilting modules over preprojective algebras of Dynkin type, Math. Z. 277 (2014), no. 3-4, 665-690.

[16] Nathan Reading, Noncrossing arc diagrams and canonical join representations, SIAM J. Discrete Math. 29 (2015), no. 2, 736-750.

[17] Sverre O. Smalø, Torsion theories and tilting modules, Bull. London Math. Soc. 16 (1984), no. $5,518-522$.

Emily Barnard, Department of Mathematical Sciences, DePaul University, 2320 N. Kenmore Ave., Suite 502, Chicago, IL 60614, USA

E-mail : e.barnard@depaul.edu

Andrew Carroll, 3778 Keating St. San Diego, CA 92110, USA

E-mail : antcarro@gmail.com

ShiJIE ZHU, Mathematics Department, University of Iowa, 14 MacLean Hall, Iowa City, IA 52242, USA

E-mail : shijie-zhu@uiowa.edu 